\title{
Non-supersymmetric F-theory compactifications on Spin(7) manifolds
}

\author{
Federico Bonetti, Thomas W. Grimm and Tom G. Pugh \\ Max Planck Institute for Physics, \\ Föhringer Ring 6, 80805 Munich, Germany \\ E-mail: bonetti@mpp.mpg.de, grimm@mpp.mpg.de, pught@mpp.mpg.de
}

ABSTRACT: We propose a novel approach to obtain four-dimensional effective actions coupled to supersymmetry-breaking boundaries by considering F-theory on manifolds with special holonomy Spin(7). To perform such studies we suggest that a duality relating Mtheory on a certain class of Spin(7) manifolds with F-theory on the same manifolds times an interval exists. The Spin(7) geometries under consideration are constructed as quotients of elliptically fibered Calabi-Yau fourfolds by an anti-holomorphic and isometric involution. The three-dimensional minimally supersymmetric effective action of M-theory on a general Spin(7) manifold with fluxes is determined and specialized to the aforementioned geometries. This effective theory is compared with an interval Kaluza-Klein reduction of a non-supersymmetric four-dimensional theory with definite boundary conditions for all fields. Using this strategy a minimal set of couplings of the four-dimensional low-energy effective actions is obtained in terms of the $\operatorname{Spin}(7)$ geometric data. We also discuss briefly the string interpretation in the Type IIB weak coupling limit.

KeYwords: F-Theory, M-Theory, String Duality

ARXIV EPRINT: 1307.5858 


\section{Contents}

1 Introduction 1

2 Geometries with Spin(7) holonomy for F-theory 4

2.1 Constructing $\operatorname{Spin}(7)$ manifolds from Calabi-Yau fourfolds 5

$\begin{array}{lll}2.2 & \operatorname{Spin}(7) \text { manifolds from Calabi-Yau elliptic fibrations } & 7\end{array}$

3 M-theory on $\operatorname{Spin}(7)$ spaces and Calabi-Yau quotients 10

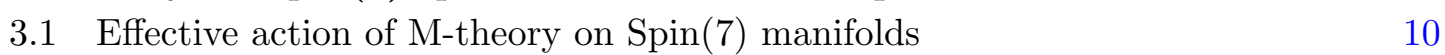

3.2 Effective action of M-theory on Spin(7) manifolds from Calabi-Yau quotients 14

3.3 Effective action of M-theory on $\operatorname{Spin}(7)$ quotients of elliptically fibered $\begin{array}{ll}\text { Calabi-Yau fourfolds } & 17\end{array}$

4 F-theory on Spin(7) manifolds $\quad 19$

4.1 Dimensional reduction of the 4d theory on an interval 20

4.2 Effective action of F-theory on Spin(7) manifolds 23

5 Comments on weak coupling and charged matter $\quad 25$

$\begin{array}{ll}5.1 \text { Weak coupling interpretation } & 26\end{array}$

$\begin{array}{lll}5.2 & \text { Aspects of charged matter } & 28\end{array}$

$\begin{array}{lll}6 & \text { Conclusions } & 30\end{array}$

$\begin{array}{ll}\text { A Conventions } & 32\end{array}$

B Example Spin(7) holonomy manifolds 33

B.1 A hypersurface in a $\mathbb{P}_{2,3,1}$ fibration of $\mathbb{P}_{1,1,1,1}$

B.2 A complete intersection in a $\mathbb{P}_{1,1,1,1}$ fibration of $\mathbb{P}_{1,1,2,2}$

\section{Introduction}

Over the last decades four-dimensional (4d) supersymmetric effective theories arising in string compactifications have been studied intensively. Minimally supersymmetric theories are considered as providing interesting physics beyond the Standard Model. Therefore it has been a crucial long-standing task to embed supersymmetric extensions of the Standard Model or Grand Unified Theories into string theory as reviewed, for example, in [1-5]. The established approach is to consider compactifications of string theory on manifolds with special holonomy, such that some of the underlying ten-dimensional (10d) supersymmetries are preserved in four dimensions and allow a supersymmetric effective theory to be determined. Precisely these supersymmetry-preserving geometries are also mathematically 
best studied and many powerful tools have been developed exploiting the interplay of geometry and low-energy physics. In this work we will examine whether one can find a rich set of string compactifications with non-supersymmetric $4 \mathrm{~d}$ effective theories, and possibly interesting phenomenological properties, while still allowing the virtues of the remarkable mathematical tools developed for special holonomy manifolds to be used.

Our considerations are based on the study of F-theory compactifications to four dimensions. Recall that F-theory vacua describe the geometry of Type IIB string compactifications with varying complexified string coupling constant. This change of coupling is encoded by the complex structure of an auxiliary two-torus, which varies over the tendimensional space-time of the Type IIB theory. Vacua of F-theory are thus torus fibrations over some base space that provides the hidden compact dimensions of Type IIB string theory. This implies that F-theory compactifications to four space-time dimensions require an eight-dimensional compact and torus-fibered geometry to be specified. Furthermore, singularities of this fibration indicate the presence of space-time filling seven-branes. Therefore, this setup geometrizes many aspects of open string physics and hence allows the construction of many interesting phenomenological models.

Minimal supersymmetry, for which the $4 \mathrm{~d}$ effective theory has four real supercharges, is preserved by the geometry if the compact eight-dimensional space has $\mathrm{SU}(4)$ holonomy, i.e. is a Calabi-Yau fourfold $[6,7]$. However, on eight-dimensional manifolds the classification by Berger [8] shows that $\mathrm{SU}(4)$ is not the maximal possible special holonomy group within the local Lorentz group $\mathrm{SO}(8)$. This maximal special holonomy group is instead given by $\operatorname{Spin}(7)$. In what follows we will refer to these manifolds with $\operatorname{Spin}(7)$ holonomy as simply Spin(7) manifolds. For these geometries one therefore is led to ask:

(1) Is there a controlled construction of $\operatorname{Spin}(7)$ manifolds that can serve as backgrounds for F-theory?

(2) What are the characteristics of the $4 \mathrm{~d}$ non-supersymmetric effective theories arising from F-theory compactifications on such Spin(7) manifolds?

(3) What is the weak coupling Type IIB string interpretation of these theories?

In this work we will attempt to systematically address these questions. It should be noted that the consideration of F-theory on Spin(7) manifolds was already mentioned in the original paper by Vafa [6], in connection with the proposals of Witten [9, 10]. However, this link has not be concretized since then. With the recent progress on deriving the $4 \mathrm{~d}$ supersymmetric effective action of F-theory on Calabi-Yau fourfolds [11], we are now endowed with the necessary advances to suggest a concrete F-theory and string theory construction.

Before even entering any analysis of the effective action, we have to answer the question of whether or not there are suitable Spin(7) manifolds that can be used for F-theory. In particular, it will be crucial to single out geometries that have an appropriate torus fibration structure to identify the F-theory compactification as a Type IIB string background. In building these manifolds we will be motivated by the constructions described by Joyce [12]. These constructions begin by considering a Calabi-Yau fourfold which is then quotiented in such a way that a $\operatorname{Spin}(7)$ manifold is generated. Here we will investigate whether this 
process, carried out for elliptically fibered Calabi-Yau fourfolds, may generate appropriate Spin(7) manifolds for use in these F-theory compactifications. It should be stressed that one expects that there exist many more examples of Spin(7) geometries that are not based on any Calabi-Yau fourfold. Definite statements about these more general cases turn out to be hard to extract, nevertheless various results of our analysis may well extend beyond the context that we consider. Importantly, these constructions based upon Calabi-Yau quotients give us control over the setup and allow our intuition about Calabi-Yau fourfold compactifications of F-theory to be used. Other explicit constructions of Spin(7) geometries appeared in $[13,14]$.

To derive effective physics of these F-theory compactifications it will be necessary to take a detour via M-theory. This can be traced back to the fact that there is no fundamental low-energy effective action of F-theory. M-theory has eleven-dimensional (11d) supergravity as a low-energy effective action [15] and hence provides a well-defined setup to study compactifications on smooth compact geometries. In fact, if one considers Mtheory on a $\operatorname{Spin}(7)$ geometry one obtains a three-dimensional (3d) effective theory with minimal supersymmetry, i.e. two supercharges [16]. ${ }^{1}$ We determine the 3 d effective action of M-theory on a general Spin(7) manifold with probe fluxes extending and applying earlier works [17-22] and determine the couplings in terms of the geometric data of the $\operatorname{Spin}(7)$ geometry. To take the F-theory limit of this $3 \mathrm{~d}$ theory to four space-time dimensions we propose the following duality:

$$
M \text {-theory on Spin(7) manifold } \cong F \text {-theory on }\left\{\begin{array}{c}
\text { Spin(7) manifold } \\
\text { (with vanishing fiber) }
\end{array} \times \text { Interval }\right\} \text {. }
$$

In the context of this work we can only make this claim in cases where the Spin(7) manifold is constructed from a Calabi-Yau fourfold as described in the previous paragraph. The extension of this to more general geometries remains an intersting open problem.

To provide evidence for (1.1) we consider a certain non-supersymmetric $4 \mathrm{~d}$ theory on an interval. If the interval is small, we perform a Kaluza-Klein reduction to an effective $3 \mathrm{~d}$ theory. Specifying the definite boundary conditions for the various fields, we argue that the $3 \mathrm{~d}$ effective theory of the zero modes is minimally supersymmetric and can be identified with the effective theory arising from a compactification of M-theory on a Spin(7) manifold. The original $4 \mathrm{~d}$ theory should be recovered in the limit in which the interval length is sent to infinity. This should correspond to sending the fiber volume of the Spin(7) manifold to zero and provide a realization of the M-theory to F-theory limit. However, in this work we will mostly consider a finite size interval either in the derivation of the $3 \mathrm{~d}$ effective theory, or in the $4 \mathrm{~d}$ lift to an effectively non-supersymmetric theory due to boundary effects.

One difference to the M-theory to F-theory limit for Calabi-Yau fourfolds is the appearance of an interval instead of a circle. This interval is crucial as the boundary conditions that are imposed project out half of the zero mode degrees of freedom that would arise in the circle reduction of a $4 \mathrm{~d}$ fermion. This means that on the level of $3 \mathrm{~d}$ zero modes only

\footnotetext{
${ }^{1}$ These compactifications are in fact on warped backgrounds, but we will not consider the impact of warping in this work.
} 
11d M-theory
effective action

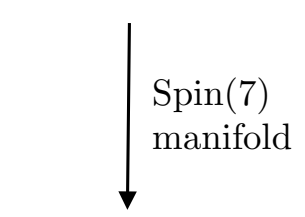

$3 \mathrm{~d} \mathcal{N}=1$

effective action 4d F-theory

effective action

interval

comparison

$3 \mathrm{~d} \mathcal{N}=1$

effective action

Figure 1. Summary of the effective actions considered in this work. The left column corresponds to the M-theory side of the duality (1.1), while the right column corresponds to the F-theory side. The comparison between the $3 \mathrm{~d} \mathcal{N}=1$ theories is performed in the case in which the $\operatorname{Spin}(7)$ manifold arises as an anti-holomorphic quotient of an elliptically fibered Calabi-Yau fourfold. We consider a fibration structure that yields a simple non-Abelian gauge group. The match of $3 \mathrm{~d}$ actions is carried out in the Coulomb branch at the level of zero modes.

a part of the $4 \mathrm{~d}$ fermionic degrees of freedom have to be completed with bosonic counterparts. This allows a non-supersymmetric spectrum in four dimensions to be dimensionally reduced to a minimally supersymmetric zero mode spectrum in three dimensions. The appearance of an interval is also natural from the construction of $\operatorname{Spin}(7)$ manifolds that we have mentioned above for which the quotient of the fourfold may be associated with the quotient of the circle that gives rise to the interval. It is crucial in (1.1) that the core features of the non-supersymmetric theory in four dimensions and the boundary conditions for the interval are fixed by the $\operatorname{Spin}(7)$ geometry.

In this work we will provide evidence for (1.1) in the context of the above mentioned quotiented Calabi-Yau geometries, and discuss important parts of the $3 \mathrm{~d}$ and $4 \mathrm{~d}$ effective actions of M-theory and F-theory. A schematic picture of the effective actions considered in the following sections can be found in figure 1. We also comment on supersymmetry restoration, which is further studied in [23]. Let us stress that it would be interesting to perform such an analysis for other classes of Spin(7) manifolds with an appropriate fibration structure.

\section{Geometries with $\operatorname{Spin}(7)$ holonomy for F-theory}

To set the stage for the discussions that follow we first recall some facts about $\operatorname{Spin}(7)$ manifolds and their construction. In subsection 2.1 we give a brief introduction to aspects of the differential and algebraic geometry of Spin(7) manifolds. We also describe the construction of $\operatorname{Spin}(7)$ manifolds as anti-holomorphic quotients of Calabi-Yau fourfolds. This construction is applied to elliptically fibered Calabi-Yau fourfolds in subsection 2.2. We discuss the fiber structures which arise and comment on seven-brane configurations that can appear. 


\subsection{Constructing Spin(7) manifolds from Calabi-Yau fourfolds}

Let us briefly recall certain important features of the geometry of $\operatorname{Spin}(7)$ holonomy eightdimensional manifolds, which we will refer to as Spin(7) manifolds. To do this it is convenient to begin by analyzing the set of independent covariantly constant spinors that may exist on such a space $Z_{8}$. All spinors on $Z_{8}$ will transform as definite representations of the holonomy group and so their properties may be studied by decomposing the representations of $\mathrm{SO}(8)$ (the holonomy group of an orientable eight-dimensional Riemannian manifold) under Spin(7). In doing this we find that the representations corresponding to Majorana-Weyl spinors decompose as

$$
\mathbf{8}_{+} \rightarrow \mathbf{1} \oplus \mathbf{7}, \quad \text { and } \quad \mathbf{8}_{-} \rightarrow \mathbf{8} .
$$

The singlets present in this decomposition determine the covariantly constant spinors of $Z_{8}$ so a given $\operatorname{Spin}(7)$ manifold may have only one independent covariantly constant spinor which we will call $\eta$. From this spinor we may construct the covariantly constant nowherevanishing $p$-forms of $Z_{8}$ by taking contractions with the gamma matrices in the usual way. However as $\eta$ is Majorana-Weyl with positive chirality the only non trivial $p$-form that may be constructed is a self-dual four-form

$$
\Phi_{m n r s}=\bar{\eta} \gamma_{m n r s} \eta, \quad \text { where } \quad \frac{1}{\hat{\mathcal{V}}} \int_{Z_{8}} \Phi \wedge \Phi=\|\Phi\|^{2}=\frac{1}{4 !} \Phi_{m n r s} \Phi^{m n r s},
$$

and where $\hat{\mathcal{V}}$ is the volume of $Z_{8}$. This four-form then gives the Cayley calibration of $Z_{8}$. We note here that by using Fierz identities one may show that $\Phi$ satisfies the useful identity

$$
\Phi^{m n p t} \Phi_{q r s t}=\frac{3}{7}\|\Phi\|^{2} \delta_{[q}^{m} \delta_{r}^{n} \delta_{s]}^{p}-\frac{9}{\sqrt{14}}\|\Phi\| \delta_{[q}^{[m} \Phi_{r s]}{ }^{n p]} .
$$

In a similar way one may analyze the cohomology of the Spin(7) manifold by decomposing the various cohomology groups under $\operatorname{Spin}(7)$. This then gives [24]

$$
\begin{array}{ll}
H^{0}\left(Z_{8}, \mathbb{R}\right)=\mathbb{R}, & H^{1}\left(Z_{8}, \mathbb{R}\right)=0, \quad H^{2}\left(Z_{8}, \mathbb{R}\right)=H_{\mathbf{2 1}}^{2}\left(Z_{8}, \mathbb{R}\right), \\
H^{3}\left(Z_{8}, \mathbb{R}\right)=H_{\mathbf{4 8}}^{3}\left(Z_{8}, \mathbb{R}\right), & H^{4}\left(Z_{8}, \mathbb{R}\right)=H_{\mathbf{1} \mathrm{S}}^{4}\left(Z_{8}, \mathbb{R}\right) \oplus H_{\mathbf{2 7} \mathrm{S}}^{4}\left(Z_{8}, \mathbb{R}\right) \oplus H_{\mathbf{3 5} \mathrm{A}}^{4}\left(Z_{8}, \mathbb{R}\right),
\end{array}
$$

where $\mathrm{S}$ and $\mathrm{A}$ indicate the self-duality and anti-self-duality of the four-forms respectively. The only in-equivalent representative of $H_{1 \mathrm{~S}}^{4}\left(Z_{8}, \mathbb{R}\right)$ is then given by $\Phi$. The Betti numbers $b^{n}\left(Z_{8}\right)=\operatorname{dim}\left(H^{n}\left(Z_{8}, \mathbb{R}\right)\right)$ satisfy one constraint,

$$
b^{2}\left(Z_{8}\right)-b^{3}\left(Z_{8}\right)-b_{S}^{4}\left(Z_{8}\right)+2 b_{A}^{4}\left(Z_{8}\right)+25=0 .
$$

This implies that there are three independent Betti numbers, for example, $b^{2}\left(Z_{8}\right), b^{3}\left(Z_{8}\right)$ and $b_{A}^{4}\left(Z_{8}\right)$.

By contrast a Calabi-Yau fourfold $Y_{4}$ has both a covariantly constant $(1,1)$ Kähler form $J$ and a holomorphic $(4,0)$ form $\Omega$. In [12] these are related to a self-dual four-form $\Phi$ by considering an anti-holomorphic and isometric involution $\sigma: Y_{4} \rightarrow Y_{4}$, i.e. $\sigma$ satisfies

$$
\sigma^{2}=\mathbb{1}, \quad \begin{cases}\text { isometric } & \sigma^{*}(g)=g, \\ \text { anti-holomorphic } & \sigma^{*}(I)=-I,\end{cases}
$$


where $g$ and $I$ are the metric and complex structure on $Y_{4}$, respectively. These conditions translate to the forms $J$ and $\Omega$ as

$$
\sigma^{*} J=-J, \quad \sigma^{*} \Omega=e^{2 i \theta} \bar{\Omega},
$$

where $\theta$ is some constant phase factor. The forms $J$ and $\Omega$ then naturally define a $\operatorname{Spin}(7)$ structure on $Y_{4}$ with $\Phi$ given by

$$
\Phi=\frac{1}{\mathcal{V}^{2}}\left(\frac{1}{\|\Omega\|} \operatorname{Re}\left(e^{-i \theta} \Omega\right)+\frac{1}{8} J \wedge J\right), \quad \text { where } \quad \mathcal{V}=\frac{1}{4 !} \int_{Y_{4}} J^{4},
$$

is the volume of $Y_{4}$ and $\|\Omega\|$ is defined analogously to (2.2). The derivation of the precise prefactors in front of $\operatorname{Re}\left(e^{-i \theta} \Omega\right)$ and $J \wedge J$ will be presented in section 3.2. The four-form $\Phi$ is invariant under the involution $\sigma$ and an associated $\operatorname{Spin}(7)$ manifold may then be constructed by quotienting $Y_{4}$ by $\sigma$ and resolving the singularities in a $\operatorname{Spin}(7)$ compatible way [12]. In this way $Y_{4}$ represents the double cover of $Z_{8}$ which relates the volumes as $\mathcal{V}=2 \hat{\mathcal{V}}$.

In preparation for the application to F-theory let us comment further on the involved geometries. We note that when considering F-theory on a Calabi-Yau space $Y_{4}^{s}$, the space can be chosen to be singular. The singularities arise, for example, when the $4 \mathrm{~d}$ theory has to have a non-Abelian gauge group. These non-Abelian singularities can be resolved in a way that is compatible with the Calabi-Yau condition to yield a manifold $Y_{4}$. We denote the anti-holomorphic involution on the singular space $Y_{4}^{s}$ by $\sigma^{s}$ and on the resolved space by $\sigma$. The respective quotient spaces are denoted by $Z_{8}^{s}=Y_{4}^{s} / \sigma^{s}$ and $Z_{8}=Y_{4} / \sigma$. The $\operatorname{Spin}(7)$ resolution of $Z_{8}$ will be denoted by $\hat{Z}_{8}$. By analogy with the standard M-theory/Ftheory duality we thus expect that the duality (1.1) relates F-theory compactified on $Z_{8}^{s}$ with M-theory compactified on $\hat{Z}_{8}$. It should be stressed that finding a resolution of $Z_{8}$ admitting a $\operatorname{Spin}(7)$ structure is a hard task and involves constructing local real $\operatorname{Spin}(7)$ ALE geometries that can be used to resolve possible orbifold singularities [12]. The Betti numbers of the resolved space can be computed as described in [12]. A stringy computation of the Betti numbers on the quotient geometry $Z_{8}$ can be found in [25]. In this work we will not be concerned with this real resolution $\hat{Z}_{8}$, and mostly work with $Z_{8}$ neglecting possible singularities. We will refer to the $\operatorname{Spin}(7)$ manifold $Z_{8}$ constructed in this way as a quotient torus fibration. Our goal is, however, to formulate the results in a general $\operatorname{Spin}(7)$ language such that they can be equally applied to the resolved geometries $\hat{Z}_{8}$. We summarize the relevant geometries in figure 2 .

The construction that is carried out in [12] assumes certain additional properties of the orbifold singularities that are required for the Spin(7) ALE resolutions which are considered there to be applied. One such condition is that the singularities introduced by quotienting with respect to $\sigma$ must be isolated points in $Z_{8}$ which lie at points that are already holomorphic orbifold singularities of $Y_{4}$. However it is anticipated that these resolution methods are by no means the only possibility. Therefore, in what follows, we will not limit ourselves to considering only the sorts of singularities which are required in [12], but will bear in mind these additional constraints. The analysis of the more general resolutions that would then be required and the physics associated with their structure will not be discussed here and therefore represents an important topic for consideration in future work. 


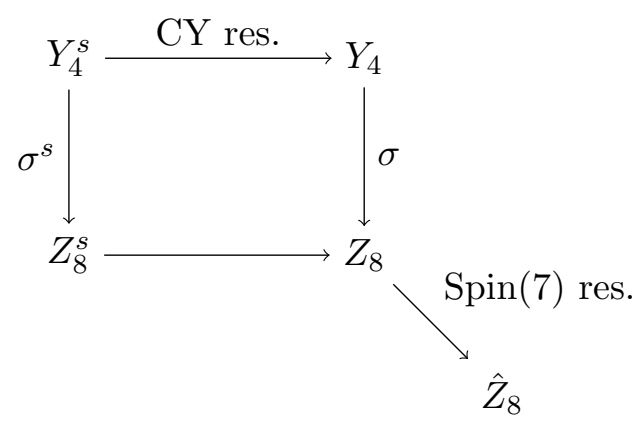

Figure 2. Construction of $\operatorname{Spin}(7)$ manifolds by using Calabi-Yau fourfolds with anti-holomorphic involutions.

\section{2 $\operatorname{Spin}(7)$ manifolds from Calabi-Yau elliptic fibrations}

In order that the $\operatorname{Spin}(7)$ manifold $Z_{8}$ can be used as a background of F-theory we require that the Calabi-Yau fourfold $Y_{4}$ is an elliptic fibration with Kähler base $B_{3}$. The elliptic fiber $\mathcal{C}_{p}$ at a point $p$ on $B_{3}$ can always be described by a Weierstrass equation ${ }^{2}$

$$
\mathcal{C}_{p}: \quad y^{2}=x^{3}+f x z^{4}+g z^{6},
$$

where $x, y, z$ are projective coordinates in $\mathbb{P}_{2,3,1}^{2}$ and $f, g$ depend on the location $p$. Away from singular points on the base, $f(u), g(u)$ are holomorphic in the complex base coordinates $u$. When the elliptic curve becomes singular, the discriminant given by

$$
\Delta=4 f^{3}+27 g^{2}
$$

vanishes. The vanishing of this function describes complex co-dimension one space in $B_{3}$ and determines the location of the space-time filling seven-branes on $B_{3}$.

Recall that F-theory on an elliptically fibered Calabi-Yau fourfold yields minimally supersymmetric theory in four space-time dimensions. ${ }^{3}$ After quotienting by the involution $\sigma$ and carrying out the resolution supersymmetry will be broken by the geometry.

The involutive symmetry $\sigma$ on the elliptic fibration is demanded to have a definite action on $B_{3}$, i.e. $\sigma$ is compatible with the fibration and induces a well-defined action on the base that we also denote by $\sigma$ for simplicity. In a given local patch $U$ on $B_{3}$ described by the coordinates $\left(z_{1}, z_{2}, z_{3}\right)$ this action can be of different types with differing dimension of the fixed space $L_{\sigma}(U) \subset U$. For example, one has

$$
\begin{array}{ll}
\left(z_{1}, z_{2}, z_{3}\right) \rightarrow\left(\bar{z}_{1}, \bar{z}_{2}, \bar{z}_{3}\right), & \Rightarrow L_{\sigma}(U) \text { is a real three-dimensional subspace of } U, \\
\left(z_{1}, z_{2}, z_{3}\right) \rightarrow\left(\bar{z}_{2},-\bar{z}_{1}, \bar{z}_{3}\right), & \Rightarrow L_{\sigma}(U) \text { is a real one-dimensional subspace of } U, \\
\left(z_{1}, z_{2}, z_{3}\right) \rightarrow\left(\frac{\bar{z}_{2}}{\bar{z}_{3}},-\frac{\bar{z}_{1}}{\bar{z}_{3}},-\frac{1}{\bar{z}_{3}}\right), & \Rightarrow L_{\sigma}(U) \text { is empty and } \sigma \text { is freely acting on } U .
\end{array}
$$

\footnotetext{
${ }^{2}$ The precise statement is that every elliptic curve is bi-rationally equivalent to such a Weierstrass equation.

${ }^{3}$ In fact, one could study the theory on the space $Y_{4}$ obtained by resolving the orbifold singularities of $Y_{4}^{\mathrm{s}}$ in a way compatible with the Calabi-Yau condition and the elliptic fibration.
} 


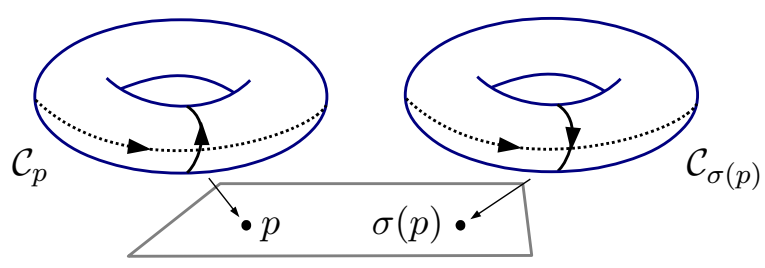

Figure 3. Generic torus fibers exchanged by the anti-holomorphic involution.

After taking the quotient the fixed space on $Y_{4}$ will represent an orbifold singularity of $Z_{8}$ which must be resolved when moving to $\hat{Z}_{8}$. If $L_{\sigma}\left(B_{3}\right)$ is one dimensional then $\sigma$ is only an involution if $B_{3}$ already has an identification under the holomorphic orbifold action generated by $\sigma^{2}$. This will have a real two-dimensional fixed space that will be associated with an additional orbifold singularity over the base that must also be resolved after the quotient.

The fixed space of $Y_{4}$, which we will call $L_{\sigma}\left(Y_{4}\right)$, can have components that are either 0,2 or 4 real dimensional or $\sigma$ can be freely acting. To investigate the action of $\sigma$ on $Y_{4}$ further we must analyze several cases which are distinguished by the location of the point $p$ on $B_{3}$ :

(1) $p \notin L_{\sigma}\left(B_{3}\right)$ : for each point $p$ on $B_{3}$ that is not a fixed point of $\sigma$ the corresponding elliptic curve $\mathcal{C}_{p}$ is mapped onto another elliptic curve $\mathcal{C}_{\sigma(p)}$ over the image point $\sigma(p)$. However, since $\sigma$ is anti-holomorphic the orientations of $\mathcal{C}_{\sigma(p)}$ and $\sigma\left(\mathcal{C}_{p}\right)$ will differ. In this case $\sigma$ will be freely acting on all points of $Y_{4}$ that project to $p$ or $\sigma(p)$, see figure 3 .

(2) $p \in L_{\sigma}\left(B_{3}\right)$ and $\Delta(p) \neq 0$ : if a point $p$ on $B_{3}$ is a fixed point of $\sigma$ the elliptic curve over this point will be mapped to itself. In particular, this implies that if $p$ is not on a seven-brane that a smooth two-torus is mapped onto itself. Recall that the fixed point set of an anti-holomorphic involution on a smooth complex two-torus either consists of up to two real lines or is empty.

(2.1) If the torus is fixed point free this implies that each point on $Y_{4}$ that projects to $p$ is actually not fixed by $\sigma$ and hence does not give rise to a singularity of $Z_{8}$. This means that $\sigma$ will be freely acting on all points of $Y_{4}$ that project to $p$. If $L_{\sigma}\left(B_{3}\right)$ is one-dimensional then the additional singularities associated with the $\sigma^{2}$ identification can be resolved in the standard toric way. Interestingly, if $\sigma$ is fixed point free on the torus but not on the base then the quotient fiber at such $p$ is a Klein bottle, see figure 4 .

(2.2) If the torus has a fixed line on it then the dimension of $L_{\sigma}\left(Y_{4}\right)$ may be up to one greater than the dimension of $L_{\sigma}\left(B_{3}\right)$, depending on the dimension of the subspace of $L_{\sigma}\left(B_{3}\right)$ over which the fixed space on the torus is a line. Since $L_{\sigma}\left(Y_{4}\right)$ 


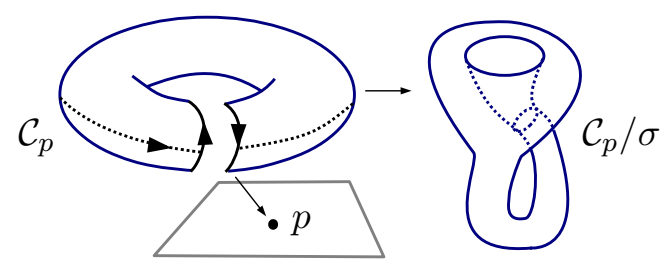

Figure 4. Fiber modded by anti-holomorphic involution to Klein bottle fibers.

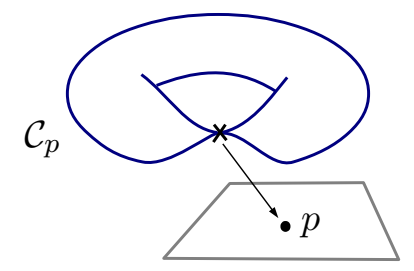

Figure 5. Nodal fiber at fixed point $p$. Involution fixes pinch-point.

must then have even dimensions greater than one, it must have dimension of either 2 or 4 . The quotient of the elliptic curve by $\sigma$ then gives rise to a cylinder. ${ }^{4}$

(3) $p \in L_{\sigma}\left(B_{3}\right)$ and $\Delta(p)=0$ : the most interesting case is if a point $p$ on $B_{3}$ is both a fixed point of $\sigma$ and lies on a seven-brane. In this case $\mathcal{C}_{p}$ is actually a singular curve. There are various possibilities for such singular curves and a systematic study should investigate all possible anti-holomorphic involutions and their fixed points. Here, let us only consider the simplest case where $\mathcal{C}_{p}$ is a nodal curve ( $I_{1}$ type), as schematically depicted in figure 5. In this case there can exist an involution $\sigma$ that has one fixed point exactly at the node of the elliptic curve. One can think of this nodal point as arising by shrinking the real one-dimensional fixed point set of an anti-holomorphic involution on a smooth elliptic curve. In this case the dimension of $L_{\sigma}\left(Y_{4}\right)$ may be an even integer less than the dimension of $L_{\sigma}\left(B_{3}\right)$, so it can be either 0 or 2 .

From this we see that if the action of $\sigma$ on $Y_{4}$ is to be fixed point free then it can have only points for which situations (1) or (2.1) apply. Alternatively if we restrict the fixed space to consist only of isolated fixed points, which is imposed in [12], then we find that situation (3) must apply in which the torus is pinched at these points. In addition to this if we also wish to consider fixed points which are already holomorphic orbifold singularities of $Y_{4}$, as is also imposed in [12], then we find that $L_{\sigma}\left(B_{3}\right)$ must be one-dimensional. An example of a space which has singularities of this sort is shown in appendix B.2.

Let us now analyze the action of the anti-holomorphic involution $\sigma$ on the elliptic fiber. To this end, we consider the case in which the elliptic fibration is presented in Weierstrass form (2.9) and we let the anti-holomorphic involution $\sigma$ act anti-linearly on the projective coordinates of $\mathbb{P}_{2,3,1}^{2}$. Any $\sigma$ action of this type may then be brought into the form

$$
\sigma: \quad(x, y, z) \rightarrow(\bar{x}, \bar{y}, \bar{z})
$$

\footnotetext{
${ }^{4}$ We note that in certain cases an anti-holomorphic involution of a smooth torus with a one-dimensional fixed space can also yield a Möbius band.
} 
by an appropriate coordinate redefinition. Comparison between (2.9) and (2.12) reveals that, in order for the anti-holomorphic involution to be well-defined on the Calabi-Yau fourfold $Y_{4}$, the sections $f$ and $g$ have to satisfy

$$
f_{\sigma(p)}=\overline{f_{p}}, \quad g_{\sigma(p)}=\overline{g_{p}},
$$

for every $p$ on the base $B_{3}$. Recall that the modular parameter $\tau$ of the elliptic fiber is given by

$$
j(\tau)=\frac{4 \cdot(24 f)^{3}}{\Delta},
$$

where the discriminant was defined in (2.10). We conclude that for any point $p$ on the base $B_{3}$

$$
j\left(\tau_{\sigma(p)}\right)=\overline{j\left(\tau_{p}\right)}=j\left(-\overline{\tau_{p}}\right) .
$$

In the last step we have made use of the fact that the $j$-function admits a Laurent series in the variable $q=e^{2 \pi i \tau}$ with integer coefficients. In summary, we can infer that

$$
\tau_{\sigma(p)}=-\overline{\tau_{p}} \quad \text { up to } \mathrm{SL}(2, \mathbb{Z}) \text { transformations. }
$$

Note that this condition is perfectly compatible with a non-trivial holomorphic dependence of the modular parameter on the base coordinates. In particular, it can be satisfied for $\tau$ profiles with non-trivial monodromies associated to the presence of seven-branes. Only in the special case in which $\tau$ is constant over the base, as in the weak coupling limit away from orientifold planes, (2.16) enforces a reality condition on $\tau$, which has to be purely imaginary. We will comment on this further in section 5 .

\section{$3 \quad$ M-theory on $\operatorname{Spin}(7)$ spaces and Calabi-Yau quotients}

Having discussed the geometry of the Spin(7) holonomy manifolds that we wish to consider, we will now describe the effective theories which arise in the reduction of M-theory on these spaces. In subsection 3.1 we will begin this analysis by considering the reduction on general Spin(7) manifolds. Then in subsection 3.2 we will analyze how this may be related to the quotient of the effective theories that arise from compactification on Calabi-Yau fourfolds. In subsection 3.3 we will then restrict to the case where these Calabi-Yau manifolds are elliptically fibered and study the redefinitions that must be made in order to move into a frame that can be lifted to the 4d F-theory dual.

\subsection{Effective action of M-theory on Spin(7) manifolds}

The compactification of M-theory on a Spin(7) manifold $\hat{Z}_{8}$ yields a 3 d effective theory with minimal $\mathcal{N}=1$ supersymmetry. The action, to quadratic order in the fermions, for a general $3 \mathrm{~d}$ theory with $\mathcal{N}=1$ supersymmetry can always be written in the form $[26,27]$

$$
\begin{aligned}
S_{\mathcal{N}=1}^{(3)}= & \int d^{3} x e\left[\frac{1}{2} R-\frac{1}{4} \Theta_{I J} \epsilon^{\mu \nu \rho} A_{\mu}^{I}\left(\partial_{\nu} A_{\rho}^{J}+\frac{1}{3} f_{K L}{ }^{J} A_{\nu}^{K} A_{\rho}^{L}\right)-\frac{1}{2} g_{\Lambda \Sigma} \mathcal{D}_{\mu} \phi^{\Lambda} \mathcal{D}^{\mu} \phi^{\Sigma}-V(\phi)\right. \\
& -\frac{1}{2} \bar{\psi}_{\mu} \gamma^{\mu \nu \rho} D_{\nu} \psi_{r}-\frac{1}{2} g_{\Sigma \Lambda} \bar{\chi}^{\Sigma} \gamma^{\mu} \mathcal{D}_{\mu} \chi^{\Lambda}+\frac{1}{2} g_{\Sigma \Lambda} \bar{\chi}^{\Sigma} \gamma^{\mu} \gamma^{\nu} \psi_{\mu} \mathcal{D}_{\nu} \phi^{\Lambda} \\
& \left.-\frac{1}{2} F \bar{\psi}_{\mu} \gamma^{\mu \nu} \psi_{\nu}+\partial_{\Lambda} F \bar{\psi}_{\mu} \gamma^{\mu} \chi^{\Lambda}+\frac{1}{2}\left(g_{\Sigma \Lambda} F-2 D_{\Sigma} \partial_{\Lambda} F+2 X_{\Sigma}^{I} X_{\Lambda}^{J} \Theta_{I J}\right) \bar{\chi}^{\Sigma} \chi^{\Lambda}\right],
\end{aligned}
$$


with covariant derivatives and scalar potential given by

$$
\mathcal{D}_{\mu} \phi^{\Lambda}=\partial_{\mu} \phi^{\Lambda}+\Theta_{I J} X^{I \Lambda} A_{\mu}^{I}, \quad V(\phi)=2 g^{\Lambda \Sigma} \partial_{\Lambda} F \partial_{\Sigma} F-4 F^{2} .
$$

Here $X^{I \Lambda}$ is the Killing vector of the target space symmetry that is gauged via (3.2). The action (3.1) contains the $\phi^{\Lambda}$-dependent metric $g_{\Lambda \Sigma}(\phi)$ that is non-degenerate and positive definite. The coefficient $\Theta_{I J}$ of the Chern-Simons term is symmetric in $I, J$, and constant which ensures the gauge invariance of the action. This represents the embedding tensor for the $3 \mathrm{~d}$ gauged supergravity theory. The real function $F(\phi)$ depends on the scalars $\phi^{\Lambda}$ and is required to satisfy $\Theta_{I J} X^{I \Lambda} \partial_{\Lambda} F=0$ for gauge invariance.

For smooth $\operatorname{Spin}(7)$ geometries $\hat{Z}_{8}$ the $\mathcal{N}=1$ vacua where studied in [16, 17, 28, 29]. The $3 \mathrm{~d}$ effective theory can be derived by reducing the action for $11 \mathrm{~d}$ supergravity [15], the bosonic part of which at lowest order in derivatives is given by

$$
S^{(11)}=\int \frac{1}{2} R * 1-\frac{1}{4} G_{4} \wedge * G_{4}-\frac{1}{12} C_{3} \wedge G_{4} \wedge G_{4}
$$

as discussed in $[17,18,20,22]$. In the full reduction one must also take into account the higher derivative terms along with the tadpole cancellation condition which for backgrounds without M2-branes becomes

$$
\frac{\chi\left(\hat{Z}_{8}\right)}{24}=\frac{1}{2} \int_{\hat{Z}_{8}} G_{4} \wedge G_{4}
$$

We will describe this reduction in the following and reconsider some aspects of the derivation presented in [22]. We stress that this reduction is actually a warped compactification, and we will neglect this back-reaction in the following leading order analysis.

We carry out the reduction by decomposing the metric and three-form of $11 \mathrm{~d}$ supergravity as

$$
d s^{2}=g_{\mu \nu} d x^{\mu} d x^{\nu}+g_{m n} d y^{m} d y^{n}, \quad \quad C_{3}=A^{I} \wedge \omega_{I}
$$

where $g_{m n}$ is the metric on $\hat{Z}_{8}$ and $\omega_{I}$ form a basis for $H^{2}\left(\hat{Z}_{8}, \mathbb{R}\right)$ with $I=1, \ldots, b^{2}\left(\hat{Z}_{8}\right)$. We will restrict to the case of $b^{3}\left(\hat{Z}_{8}\right)=0$ for simplicity. The $3 \mathrm{~d}$ theory will then admit $\mathrm{U}(1)$ gauge symmetries associated with the vectors $A^{I}$.

In performing the Kaluza-Klein reduction one has to allow the metric of the internal geometry $\hat{Z}_{8}$ to vary without leaving the class of $\operatorname{Spin}(7)$ geometries. To find the permitted deformations one constructs the Lichnerowicz operator on $\hat{Z}_{8}$ and shows that its zero modes are in one-to-one correspondence with the set of anti-self-dual four-forms $\xi_{A}, A=$ $1, \ldots, b_{\mathrm{A}}^{4}\left(\hat{Z}_{8}\right)$, along with one additional zero mode that corresponds to a rescaling of the overall volume. This implies that there will be $b_{A}^{4}\left(\hat{Z}_{8}\right)+1$ real scalar fields $\varphi^{A}$ and $\hat{\mathcal{V}}$ parameterizing the deformations of the $\operatorname{Spin}(7)$ structure. The under a variation of the scalars $\hat{\mathcal{V}}$ and $\varphi^{A}$ the Cayley calibration $\Phi$ and the metric are deformed as

$$
\delta \Phi=K_{\hat{\mathcal{V}}} \Phi \delta \hat{\mathcal{V}}+\left(K_{A} \Phi+\xi_{A}\right) \delta \varphi^{A}, \quad \delta g_{m n}=\frac{1}{4 \hat{\mathcal{V}}} g_{m n} \delta \hat{\mathcal{V}}+\frac{7}{6\|\Phi\|^{2}}\left(\xi_{A}\right)_{m p q r} \Phi_{n}^{p q r} \delta \varphi^{A}
$$


where the factors in $\delta g_{m n}$ are chosen in accord with (2.3). As a result of the anti-self-duality of $\xi_{A}$, the variation of the metric with respect to $\varphi^{A}$ is symmetric and trace-free [30]. The real coefficients in (3.6) given by $K_{\hat{\mathcal{V}}}$ and $K_{A}$ are in general functions of $\hat{\mathcal{V}}$ and $\varphi^{A}$ and depend on the normalization of $\Phi$.

Upon performing the dimensional reduction, followed by a Weyl rescaling of the $3 \mathrm{~d}$ metric to move into the Einstein frame, the bosonic part of the effective action is given by $S_{\hat{Z}_{8}}^{(3)}=\int \frac{1}{2} R * 1-\frac{1}{2} h_{I J} F^{I} \wedge * F^{J}-\frac{1}{4} \Theta_{I J} A^{I} \wedge F^{J}-\frac{1}{2} g_{\hat{\mathcal{V}} \hat{\mathcal{V}}} d \hat{\mathcal{V}} \wedge * d \hat{\mathcal{V}}-\frac{1}{2} g_{A B} d \varphi^{A} \wedge * d \varphi^{B}-V(\varphi) * 1$,

where

$$
g_{\hat{\mathcal{V}} \hat{\mathcal{V}}}=\frac{9}{8} \hat{\mathcal{V}}^{-2}, \quad g_{A B}=-\frac{7}{2} \frac{\int_{\hat{Z}_{8}} \xi_{A} \wedge \xi_{B}}{\int_{\hat{Z}_{8}} \Phi \wedge \Phi}, \quad h_{I J}=\frac{1}{2 \hat{\mathcal{V}}} \int_{\hat{Z}_{8}} \omega_{I} \wedge * \omega_{J},
$$

and the scalar potential $V(\varphi)$ is of the form (3.2). This action is less general then (3.1). Firstly, we have only included Abelian vectors. More importantly, we did not dualize all dynamical vector degrees of freedom into scalar degrees of freedom as it is always possible in three dimensions. Therefore the kinetic terms of the vectors with $\varphi^{A}$-dependent metric $h_{I J}$ still appears in (3.7). Dualizing all vector degrees of freedom yields new scalars $\zeta_{I}$ with metric $h^{I J}$, the inverse of $h_{I J}$. The presence of a Chern-Simons term in (3.7) implies that the $\zeta_{I}$ are in general gauged with covariant derivative

$$
\mathcal{D} \zeta_{I}=d \zeta_{I}+\Theta_{I J} A^{J} .
$$

Hence, the action (3.7) allows us to determine all couplings in (3.1): $\phi^{\Lambda}=\left(\hat{\mathcal{V}}, \varphi^{A}, \zeta_{I}\right)$, $g_{\Lambda \Sigma}=\left(\frac{9}{8 \hat{\mathcal{V}}^{2}}, g_{A B}, h^{I J}\right)$, and $X_{J}^{I}=\delta_{J}^{I}, X^{I A}=0$.

So far we have not discussed the scalar potential $V$ and the Chern-Simons coupling $\Theta_{I J}$. In fact, in a compactification without fluxes both vanish identically. They are, however, induced if one allows for a non-trivial flux background of the field strength $d C_{3}$. Let us denote the background flux on $\hat{Z}_{8}$ by $G_{4}$. A direct reduction of $11 \mathrm{~d}$ supergravity then implies that a flux-induced Chern-Simons term takes the form

$$
\Theta_{I J}=\int_{\hat{Z}_{8}} G_{4} \wedge \omega_{I} \wedge \omega_{J}
$$

More involved is the derivation of the flux-induced scalar potential from a real function $F$. After dimensional reduction of the full action including the higher curvature term, one uses the tadpole cancellation condition (3.4) to show that the scalar potential takes the form

$$
V=\frac{1}{4 \hat{\mathcal{V}}^{3}}\left(\int_{\hat{Z}_{8}} G_{4} \wedge * G_{4}-\int_{\hat{Z}_{8}} G_{4} \wedge G_{4}\right)=-\frac{1}{2 \hat{\mathcal{V}}^{3}} \int_{\hat{Z}_{8}} G_{4}^{A} \wedge G_{4}^{A},
$$

where $G_{4}^{A}$ is the anti-self-dual part of the background flux $G_{4}$. To generally derive $F$ let us first note that it was argued in [22] that $F$ should be proportional to $\int_{\hat{Z}_{8}} G_{4} \wedge \Phi$. The factor in front of this flux integral can, however, be field-dependent. In fact the correct form of $F$ is given by

$$
F=\frac{\sqrt{7}}{4 \sqrt{2}\|\Phi\| \hat{\mathcal{V}}^{2}} \int_{\hat{Z}_{8}} G_{4} \wedge \Phi
$$


The derivatives of $F$ then satisfy

$$
\frac{\partial F}{\partial \varphi^{A}}=\frac{\sqrt{7}}{4 \sqrt{2}\|\Phi\| \hat{\mathcal{V}}^{2}} \int_{\hat{Z}_{8}} G_{4} \wedge \xi_{A}, \quad \frac{\partial F}{\partial \hat{\mathcal{V}}}=-\frac{3}{2 \hat{\mathcal{V}}} F
$$

which are independent of the precise form of $K_{\hat{\mathcal{v}}}$ and $K_{A}$ in (3.6) as these cancel when taking the derivative. ${ }^{5}$ Inserting (3.13), (3.12) and the inverse metrics $g^{A B}, g^{\hat{\mathcal{V}} \hat{\mathcal{V}}}$ obtained from (3.8) into the general form of the $\mathcal{N}=1$ scalar potential (3.2) one readily shows match with (3.11).

We conclude this section by performing a rearrangement of the $\operatorname{Spin}(7)$ moduli that will be useful in the comparison to the Calabi-Yau reduction of section 3.2. To begin with, we divide the $\operatorname{Spin}(7)$ moduli $\varphi^{A}$ into two subsets, $\varphi^{A}=\left(\varphi^{\mathcal{K}}, \varphi^{\tilde{I}_{-}}\right)$. This notation is chosen to make contact to section 3.2. Note that this partition of the Spin(7) moduli is supposed to be such that the associated anti-self-dual four-forms satisfy the orthogonality condition

$$
\int_{\hat{Z}_{8}} \xi_{\mathcal{K}} \wedge \xi_{\tilde{I}_{-}}=0
$$

Next we extend the range of the index $\tilde{I}_{-}$by defining a new index $I_{-}$that includes one additional entry and define $\phi^{I_{-}}=\left(\hat{\phi}, \hat{\phi} \varphi^{\tilde{I}_{-}}\right)$. This definition is such that that the variation of $\Phi$ in (3.6) is now given by

$$
\delta \Phi=K_{\hat{\mathcal{V}}} \Phi \delta \hat{\mathcal{V}}+\left(\mathbb{K}_{I_{-}} \Phi+\eta_{I_{-}}\right) \delta \phi^{I_{-}}+\left(K_{\mathcal{K}} \Phi+\xi_{\mathcal{K}}\right) \delta \varphi^{\mathcal{K}}
$$

where

$$
\mathbb{K}_{I_{-}}=\left(-\frac{\varphi^{\tilde{J}_{-}} K_{\tilde{J}_{-}}}{\hat{\phi}}, \frac{K_{\tilde{I}_{-}}}{\hat{\phi}}\right), \quad \eta_{I_{-}}=\left(-\frac{\varphi^{\tilde{J}_{-}} \xi_{\tilde{J}_{-}}}{\hat{\phi}}, \frac{\xi_{\tilde{I}_{-}}}{\hat{\phi}}\right)
$$

These definitions then imply the constraints

$$
\phi^{I_{-}} \mathbb{K}_{I_{-}}=0, \quad \phi^{I_{-}} \eta_{I_{-}}=0,
$$

which means that the action (3.7) develops a new local symmetry under under which

$$
\phi^{I_{-}} \rightarrow \lambda \phi^{I_{-}}, \quad \Phi \rightarrow \lambda \Phi .
$$

As anticipated above, this constrained formulation will be helpful in section 3.2. It might also be useful, however, in finding generalizations of the F-theory construction to $\operatorname{Spin}(7)$ manifolds that are not obtained as Calabi-Yau quotients.

\footnotetext{
${ }^{5}$ One can also show that given a general Cayley calibration $\Phi$, which varies as (3.6), it is possible to define an alternatively normalized self-dual four-form $\hat{\Phi}$ which is also a singlet of $\operatorname{Spin}(7)$ and satisfies

$$
\hat{\Phi}=\frac{1}{\|\Phi\| \hat{\mathcal{V}}^{2}} \Phi, \quad \hat{K}_{\hat{\mathcal{V}}}=-\frac{3}{2} \hat{\mathcal{V}}^{-1}, \quad \hat{K}_{A}=0
$$

This corresponds to the normalization for $\Phi$ chosen in (2.8). 


\subsection{Effective action of M-theory on $\operatorname{Spin}(7)$ manifolds from Calabi-Yau quo- tients}

In the following we would like to introduce $\operatorname{Spin}(7)$ geometries whose effective theories can be up-lifted to four dimensions via the M-theory to F-theory limit. It is an outstanding question to characterize such geometries generally. In order to approach this problem we therefore restrict our analysis to $\operatorname{Spin}(7)$ geometries arsing from elliptically fibered Calabi-Yau fourfolds as introduced in section 2.2. Our aim is to first show, that the $3 \mathrm{~d}$ $\mathcal{N}=2$ theories arising in Calabi-Yau fourfold compactifications of M-theory are truncated to $\mathcal{N}=1$ when performing the anti-holomorphic quotient $Y_{4} / \sigma$, with an involution $\sigma$ as in (2.7). We note that the following steps bear many similarities to the construction of $4 \mathrm{~d}$ Type IIA Calabi-Yau orientifold actions [31]. However, here we are truncating $3 \mathrm{~d} \mathcal{N}=2$ supersymmetry to $\mathcal{N}=1$ supersymmetry. ${ }^{6}$ Truncations of $\mathcal{N}=2$ Chern-Simons theories to $\mathcal{N}=1$ induced by an anti-holomorphic involution have been also considered in [33].

Let us first recall the general form of a $3 \mathrm{~d} \mathcal{N}=2$ action. The bosonic part of this can always be brought to the form

$$
S_{\mathcal{N}=2}^{(3)}=\int \frac{1}{2} R * 1-\frac{1}{4} \Theta_{I J} A^{I} \wedge\left(d A^{J}+\frac{2}{3} f_{K L}{ }^{J} A^{K} \wedge A^{L}\right)-g_{\mathcal{A} \overline{\mathcal{B}}} \mathcal{D} M^{\mathcal{A}} \wedge * \mathcal{D} \bar{M}^{\mathcal{B}}-\tilde{V} * 1
$$

where $g_{\mathcal{A} \overline{\mathcal{B}}}=\partial_{\mathcal{A}} \partial_{\overline{\mathcal{B}}} K$ is a Kähler metric and $\tilde{V}(M, \bar{M})$ is the scalar potential. This scalar potential is generally of the form

$$
\tilde{V}=e^{K}\left(K^{\mathcal{A} \overline{\mathcal{B}}} D_{\mathcal{A}} W \overline{D_{\mathcal{B}} W}-4|W|^{2}\right)+\left(K^{\mathcal{A} \overline{\mathcal{B}}} \partial_{\mathcal{A}} \mathcal{T} \overline{\partial_{\mathcal{B}} \mathcal{T}}-\mathcal{T}^{2}\right)
$$

where $W(M)$ is a holomorphic superpotential and $\mathcal{T}$ is a real potential. One may also note that in the $\mathcal{N}=2$ case the presence of a non-vanishing $\mathcal{T}$ is linked to the gaugings $\mathcal{D} M^{\mathcal{A}}$.

The $3 \mathrm{~d} \mathcal{N}=2$ effective action for a Calabi-Yau fourfold compactification of $11 \mathrm{~d}$ supergravity was derived in $[34,35]$. For the case $b^{3}\left(Y_{4}\right)=0$ it takes a particularly simple form. The reduction yields $h^{3,1}\left(Y_{4}\right)$ complex structure moduli $z^{\mathcal{K}}$, which are complex fields and parametrize the changes of the $(4,0)$-form $\Omega(z)$. In addition there are $h^{1,1}\left(Y_{4}\right)$ real Kähler structure deformations $v^{I}$ arising in the expansion of the Kähler form $J=v^{I} \omega_{I}$. The expansion of the M-theory three-form $C_{3}=A^{I} \wedge \omega_{I}$ yields $h^{1,1}\left(Y_{4}\right) 3 \mathrm{~d}$ vectors $A^{I}$. The vectors $A^{I}$ together with $v^{I}$ form the bosonic components of $3 \mathrm{~d} \mathcal{N}=2$ vector multiplets. After dualizing all dynamical vector degrees of freedom into scalars $\zeta_{I}$, the kinetic terms of the $3 \mathrm{~d} \mathcal{N}=2$ supergravity theory are encoded by a Kähler potential

$$
K(z, T)=-\log \int_{Y_{4}} \Omega \wedge \bar{\Omega}-3 \log \mathcal{V},
$$

which is evaluated as a function of the $h^{3,1}\left(Y_{4}\right)+h^{1,1}\left(Y_{4}\right)$ complex coordinates $z^{\mathcal{K}}$ and

$$
T_{I}=\frac{1}{3 !} \int_{Y_{4}} \omega_{I} \wedge J^{3}+i \zeta_{I}
$$

\footnotetext{
${ }^{6}$ A systematic study of spontaneous $\mathcal{N}=2$ to $\mathcal{N}=1$ breaking in three dimensions can be found in [32].
} 
In the presence of background fluxes $G_{4}$ a non-trivial Chern-Simons term with $\Theta_{I J}$ exactly as in (3.10) is induced. As above in (3.9) this also implies the presence of gaugings $\mathcal{D} T_{I}=$ $d T_{I}+i \Theta_{I J} A^{J}$. Furthermore, a scalar potential arises from the functions

$$
\mathcal{T}=\frac{1}{4 \mathcal{V}^{2}} \int_{Y_{4}} G_{4} \wedge J^{2}, \quad W=\int_{Y_{4}} G_{4} \wedge \Omega
$$

where $\mathcal{T}$ is in accord with the gauged shift symmetries.

In order to implement the $\mathcal{N}=1$ truncation we first note that the relevant forms have to transform under $\sigma^{*}$ as

$$
\sigma^{*} J=-J, \quad \sigma^{*}(C \Omega)=\overline{C \Omega}, \quad \sigma^{*} C_{3}=C_{3},
$$

where the first two conditions already appeared in (2.7) when inserting the definition

$$
C=e^{-i \theta} e^{K / 2},
$$

with $K$ as defined in (3.22). To perform the reduction one thus has to split the cohomology of $Y_{4}$ into parity-even and parity-odd eigenspaces as

$$
H^{n}\left(Y_{4}, \mathbb{R}\right)=H_{+}^{n}\left(Y_{4}, \mathbb{R}\right) \oplus H_{-}^{n}\left(Y_{4}, \mathbb{R}\right) .
$$

The surviving vectors in the expansion of $C_{3}$ only arise from elements of $H_{+}^{2}\left(Y_{4}\right)$, while the surviving Kähler structure scalars arise from elements of $H_{-}^{2}\left(Y_{4}\right)$. Thus, one has

$$
C_{3}=A^{I_{+}} \wedge \omega_{I_{+}}, \quad I_{+}=1, \ldots, h_{+}^{1,1}\left(Y_{4}\right), \quad J=v^{I_{-}} \omega_{I_{-}}, \quad I_{-}=1, \ldots, h_{-}^{1,1}\left(Y_{4}\right) .
$$

Applying this to the dual complex scalars $T_{I}$ introduced in (3.23) one finds the split

$$
T_{I}=\left(T_{I_{+}}, T_{I_{-}}\right)=\left(-i \operatorname{Im} T_{I_{+}}, \operatorname{Re} T_{I_{-}}\right), \quad \operatorname{Im} T_{I_{-}}=\operatorname{Re} T_{I_{+}}=0 .
$$

In other words, out of the $h^{1,1}\left(Y_{4}\right)$ complex coordinates $T_{I}$ only $h^{1,1}\left(Y_{4}\right)$ real coordinates survive in the quotient theory. Similarly, the $h^{3,1}\left(Y_{4}\right)$ complex fields $z^{\mathcal{K}}$ encoding complex structure deformations are reduced to $h^{3,1}\left(Y_{4}\right)$ real complex structure deformations $\varphi^{\mathcal{K}}$. This can be inferred by considering all complex structure deformations of $\Omega$ preserving the condition (3.25). One can chose local coordinates such that $\varphi^{\mathcal{K}}=\operatorname{Re} z^{\mathcal{K}}$. In summary, the involution truncates the $\mathcal{N}=2$ Kähler manifold spanned by $T_{I}$ and $z^{\mathcal{K}}$ to a real Lagrangian submanifold $\mathcal{L}_{\sigma}$ parametrized by $\zeta_{I_{+}}, \operatorname{Re} T_{I_{-}}$and $\varphi^{\mathcal{K}}$.

To compare these degrees of freedom which survive the quotient with those described in the $\operatorname{Spin}(7)$ reduction of subsection 3.1 it is necessary to redefine the fields. The vectors $A^{I_{+}}$and the volume $\mathcal{V}$ are simply identified with the vectors $A^{I}$ and the volume $\mathcal{V}$ in (3.7), while the $b_{A}^{4}\left(Z_{8}\right)$ scalar fields $\varphi^{A}$ in (3.7) parametrize the independent degrees of freedom of the constrained fields

$$
\phi^{\hat{A}}=\left(\varphi^{\mathcal{K}}, \phi^{I_{-}}\right), \quad \text { where } \quad \hat{A}=1, \ldots, 1+b_{A}^{4}\left(Z_{8}\right), \quad \phi^{I_{-}}=\mathcal{V}^{-\frac{1}{4}} v^{I_{-}} .
$$

They satisfy the constraint

$$
N \equiv \frac{1}{4 !} \mathcal{K}_{I_{-} J_{-} K_{-} L_{-}} \phi^{I_{-}} \phi^{J_{-}} \phi^{K_{-}} \phi^{L_{-}}=1
$$


as a result of the definition (3.30). This condition can be viewed as a gauge fixing of the additional symmetry introduced in (3.19). In terms of these fields the bosonic part of the effective theory describing the projected Calabi-Yau reduction is given by

$$
\begin{aligned}
S_{Y_{4} / \sigma}^{(3)}= & \int \frac{1}{2} R * 1-\frac{1}{2} h_{I_{+} J_{+}} F^{I_{+}} \wedge * F^{J_{+}}-\frac{1}{4} \Theta_{I_{+} J_{+}} A^{I_{+}} \wedge d A^{J_{+}}-\frac{1}{2} g \mathcal{V} \mathcal{V} \mathcal{V} \wedge * d \mathcal{V} \\
& -\frac{1}{2} \tilde{g}_{I_{-} J_{-}} d \phi^{I_{-}} \wedge * d \phi^{J_{-}}-\frac{1}{2} \tilde{g}_{\mathcal{K} \mathcal{I}} d \varphi^{\mathcal{K}} \wedge * d \varphi^{\mathcal{I}}-V * 1
\end{aligned}
$$

where the scalar metrics may be written as

$$
\begin{aligned}
g \mathcal{V V} & =\frac{9}{8} \mathcal{V}^{-2}, & h_{I_{+} J_{+}} & =\frac{1}{2 \mathcal{V}} \int_{Y_{4}} \omega_{I_{+}} \wedge * \omega_{J_{+}}, \\
\tilde{g}_{I_{-} J_{-}} & =-4 \mathcal{V}^{3} \int_{Y_{4}} \eta_{I_{-}} \wedge \eta_{J_{-}}, & \tilde{g}_{\mathcal{K} \mathcal{L}} & =-4 \mathcal{V}^{3} \int_{Y_{4}} \xi_{\mathcal{K}} \wedge \xi_{\mathcal{L}},
\end{aligned}
$$

and where

$$
\begin{aligned}
\eta_{I_{-}} & =\frac{1}{4} \mathcal{V}^{-\frac{3}{2}} P_{I_{-}}{ }^{J_{-}} \omega_{J_{-}} \wedge J_{\phi}, & P_{I_{-}}{ }^{J_{-}} & =\delta_{I_{-}}{ }^{J_{-}}-\frac{1}{4 !} \mathcal{K}_{I_{-} K_{-} L_{-} M_{-}} \phi^{K_{-}} \phi^{L_{-}} \phi^{M_{-}} \phi^{J_{-}}, \\
\xi_{\mathcal{K}} & =\operatorname{Re}\left(C \chi_{\mathcal{K}}\right), & \mathcal{K}_{I_{-} J_{-} K_{-} L_{-}} & =\int_{Y_{4}} \omega_{I_{-}} \wedge \omega_{J_{-}} \wedge \omega_{K_{-}} \wedge \omega_{L_{-}} .
\end{aligned}
$$

We have used the definition $J_{\phi}=\phi^{I_{-}} \omega_{I_{-}}$. Note that the constraint (3.31) is responsible for the projection matrices $P_{I_{-}}{ }^{J_{-}}$that appear in the definition of the scalar metric. The Chern-Simons terms in (3.32) are induced by $G_{4}$ fluxes as in (3.10) and read

$$
\Theta_{I_{+} J_{+}}=\frac{1}{2} \int_{Y_{4}} \omega_{I_{+}} \wedge \omega_{J_{+}} \wedge G_{4}
$$

By considering the potential of the truncated theory and matching this with (3.2) we see that

$$
F=e^{K / 2} \operatorname{Re} W+\frac{1}{2} \mathcal{T}=\int_{Y_{4}} G_{4} \wedge\left(\operatorname{Re}(C \Omega)+\frac{1}{8} \mathcal{V}^{-2} J \wedge J\right)
$$

By comparing this with (3.12) we may then read off $\Phi=\left(\operatorname{Re}(C \Omega)+\frac{1}{8} \mathcal{V}^{-2} J \wedge J\right)$ up to a choice of normalization. This is the expression for $\Phi$ that we already quoted in (2.8). In the remainder of this subsection we discuss the structure of the resulting Spin(7) field space in more detail.

To investigate the metric on the $\operatorname{Spin}(7)$ field space we need to determine its variations with respect to the coordinates introduced in (3.30). This again requires the constraint (3.31) to be consistently implemented. One way to achieve this is to first express $\Phi$ in terms of $\mathcal{V}$ and $N$ before taking derivatives and later impose (3.31). Concretely, one has

$$
\Phi=\frac{1}{\mathcal{V}^{3 / 2}}\left(\frac{\operatorname{Re}\left(e^{-i \theta} \Omega\right)}{\left(\int_{Y_{4}} \Omega \wedge \bar{\Omega}\right)^{1 / 2}}+\frac{1}{8} \frac{J_{\phi} \wedge J_{\phi}}{N^{1 / 2}}\right) .
$$

Then taking the variations of this with respect to $\mathcal{V}, \phi^{I_{-}}$, and $\varphi^{\mathcal{K}}$ we find

$$
\left.\delta \Phi\right|_{N=1}=-\frac{3}{2} \mathcal{V}^{-1} \Phi \delta \mathcal{V}+\eta_{I_{-}} \delta \phi^{I_{-}}+\xi_{\mathcal{K}} \delta \varphi^{\mathcal{K}},
$$


and in addition find that the normalization of $\Phi$ is such that

$$
\int_{\hat{Z}_{8}} \Phi \wedge \Phi=\frac{7}{16} \mathcal{V}^{-3}
$$

Then by comparing the variation (3.38) with (3.6) we may identify the forms $\xi_{\mathcal{K}}$ and $\eta_{I_{-}}$with the $\operatorname{Spin}(7)$ forms $\xi_{A}$. More precisely, note that the constraint (3.31) implies $\phi^{I_{-}} \eta_{I_{-}}=0$. We thus identify the coordinates $\phi^{I_{-}}$and forms $\eta_{I_{-}}$with the quantities constructed after (3.15). Moreover, we find that the projected $Y_{4}$ moduli metric (3.33) matches the Spin(7) moduli metric (3.8). As expected from the general Spin(7) analysis, $\eta_{I_{-}}$and $\xi_{\mathcal{K}}$ also form a basis for the complete set of anti-self-dual four-forms of $Y_{4}$ which are invariant under $\sigma .^{7}$

\subsection{Effective action of M-theory on $\operatorname{Spin}(7)$ quotients of elliptically fibered Calabi-Yau fourfolds}

In order to derive the $4 \mathrm{~d}$ effective action of F-theory on a Spin(7) holonomy manifold, we must now restrict our M-theory reduction of section 3.2 to be based on elliptically fibered Calabi-Yau fourfolds. In doing this we will denote the base of the elliptically fibered Calabi-Yau $Y_{4}$ by $B_{3}$. Recall that for an elliptic fibration we find in cohomology

$$
12 c_{1}\left(B_{3}\right)=[\Delta]_{B_{3}},
$$

where $[\Delta]_{B_{3}}$ is the Poincaré-dual two-form to the discriminant locus (2.10) in the base $B_{3}$. We note that both $c_{1}\left(B_{3}\right)$ and $[\Delta]_{B_{3}}$ have to transform with a negative sign under the anti-holomorphic and isometric involution $\sigma$. This requirement also ensures that $\Delta$ has a finite volume, i.e. $\int_{\Delta} J \wedge J$ does not vanish.

The two-form associated to the zero section of the elliptic fibration is denoted by $\omega_{0}$. In this work we will be only dealing with Calabi-Yau fourfold geometries with holomorphic zero sections. Note that $\omega_{0}$ must transform with a negative sign under $\sigma^{*}$. In fact, as we discussed in section 2.2 the homology class of the torus fiber is negative under $\sigma$, since $\sigma$ reverses the orientation of the two-torus. This property can also be seen by noting that the base intersects the fiber exactly once. As we will discuss later, this allows us to perform the uplift by sending the coefficient $\phi^{0}$ in the expansion of $J$ to zero.

As the involution $\sigma$ also descends to the base, the cohomology of $B_{3}$ may be decomposed under the action of $\sigma$ as $H^{p}\left(B_{3}\right)=H_{+}^{p}\left(B_{3}\right) \oplus H_{-}^{p}\left(B_{3}\right)$. This means that one can write

$$
\left(\omega_{\alpha}\right)=\left(\omega_{\alpha_{+}}, \omega_{\alpha_{-}}\right), \quad \alpha_{ \pm}=1, \ldots, h_{ \pm}^{1,1}\left(B_{3}\right),
$$

where $\omega_{\alpha_{ \pm}}$are obtained by pulling back elements of $H_{ \pm}^{2}\left(B_{3}\right)$ to $H_{ \pm}^{2}\left(Y_{4}\right)$.

We will also allow for resolved singularities of the elliptic fibration of $Y_{4}$ that correspond to simple non-Abelian gauge groups $G$ in the dual F-theory compactification on $Y_{4}$. The location of these non-Abelian singularities defines a divisor $S$ in $B_{3}$. In the simple analysis that follows we will assume that there is only one stack of seven-branes on $B_{3}$ that describe

\footnotetext{
${ }^{7}$ In fact the basis formed by $\eta_{I_{-}}$and $\xi_{\mathcal{K}}$ is complete but also degenerate as a result of the projection matrix $P_{I_{-}}{ }^{J_{-}}$which appears in the definition of $\eta_{I_{-}}$.
} 
a non-Abelian gauge group and so $S$ has only one connected component. This significant simplification by no means represents the most general setup which we will not address here. As a result the actions that follow will not represent the most general possibilities.

The Poincaré dual two form $[S]_{B_{3}}$ lifted to $Y_{4}$, admits the expansion $b_{S}^{\alpha_{-}} \omega_{\alpha-}$ defining constant coefficients $b_{S}^{\alpha_{-}}$. As noted above, $[\Delta]_{B_{3}}$ and hence $[S]_{B_{3}}$ have negative parity under $\sigma$ so only the $\omega_{\alpha-}$ appear in the expansion. The non-Abelian singularities are resolved by introducing new two-forms $\omega_{i}, i=1, \ldots, \operatorname{rank}(G)$. Assuming the absence of Abelian gauge factors one has $\operatorname{rank}(G)=h^{1,1}\left(Y_{4}\right)-h^{1,1}\left(B_{3}\right)-1$. Let us note that all $\operatorname{rank}(G)$ forms $\omega_{i}$ are in fact negative under $\sigma^{*}$. To infer this we stress that each exceptional divisor is a $\mathbb{P}^{1}$ fibration over the seven-brane locus in the base $B_{3}$. Within $B_{3}$ the seven-brane divisor $S$ and its volume form are positive under $\sigma$ by Poincaré duality. ${ }^{8}$ Since the anti-holomorphic $\sigma$ reverses the sign of the volume form of the $\mathbb{P}^{1}$-fiber, we conclude that the exceptional divisors and their Poincaré dual two-forms $\omega_{i}$ are negative under $\sigma$. In summary, we find that the two-forms representing $H^{2}\left(Y_{4}\right)$ are split according to

$$
\left(\omega_{I_{+}}\right)=\left(\omega_{\alpha_{+}}\right), \quad\left(\omega_{I_{-}}\right)=\left(\omega_{0}, \omega_{\alpha_{-}}, \omega_{i}\right) .
$$

This implies that the truncated spectrum of the $3 \mathrm{~d} \mathcal{N}=1$ theory is given by $h_{+}^{1,1}\left(B_{3}\right)$ vectors $A^{\alpha_{+}}$, and $h^{1,1}\left(Y_{4}\right)-h_{+}^{1,1}\left(B_{3}\right)+h^{3,1}\left(Y_{4}\right)$ scalars $v^{I_{-}}=\left(v^{0}, v^{\alpha_{-}}, v^{i}\right)$ and $\varphi^{\mathcal{K}}$.

One can now systematically study all intersection numbers that are not forbidden by the $\sigma$-parity. Since the volume form on $Y_{4}$ is positive under $\sigma^{*}$ the vanishing intersection numbers $\mathcal{K}_{I J K L}=\int_{Y_{4}} \omega_{I} \wedge \omega_{J} \wedge \omega_{K} \wedge \omega_{L}$ are

$$
\mathcal{K}_{I_{+} J_{+} K_{+} L_{-}}=0, \quad \mathcal{K}_{I_{+} J_{-} K_{-} L_{-}}=0 .
$$

Combined with the intersection structure on elliptic fibrations one thus finds that for the potential $\hat{K}=\left.K\right|_{\mathcal{L}_{\sigma}}$ the relevant non-vanishing intersections are

$$
\begin{aligned}
\mathcal{K}_{0 \alpha_{-} \beta_{-} \gamma_{-}} & \equiv \kappa_{\alpha_{-} \beta_{-} \gamma_{-}}, & \mathcal{K}_{0 \alpha_{-} \beta_{+} \gamma_{+}} & \equiv \kappa_{\alpha_{-} \beta_{+} \gamma_{+}}, \\
\mathcal{K}_{i j \alpha_{-} \beta_{-}} & =-C_{i j} b_{S}^{\gamma_{-}} \kappa_{\gamma_{-} \alpha_{-} \beta_{-}}, & \mathcal{K}_{i j \alpha_{+} \beta_{+}} & =-C_{i j} b_{S}^{\gamma_{-}} \kappa_{\gamma_{-} \alpha_{+} \beta_{+}},
\end{aligned}
$$

where $\kappa_{\alpha_{-} \beta_{-} \gamma_{-}}$and $\kappa_{\alpha_{-} \beta_{+} \gamma_{+}}$are the triple intersections on $B_{3}$. The matrix $C_{i j}$ is the Cartan matrix of the non-Abelian gauge group $G$. Let us stress that there are numerous other intersection numbers that are in general non-zero on $Y_{4} / \sigma$. In particular, intersection numbers involving $\left(\omega_{0}\right)^{n}, n>0$ will play a crucial role when matching the F-theory and M-theory reduction at the one-loop level $[36-38] .{ }^{9}$ Crucially, this requires a redefinition of the coordinates

$$
\hat{\phi}^{\alpha_{-}}=\phi^{\alpha_{-}}+\frac{1}{2} K^{\alpha_{-}} \phi^{0}
$$

where $-K^{\alpha_{-}}$are the coefficients of $c_{1}\left(B_{3}\right)$ in the basis $\omega_{\alpha_{-}}$[39].

The splitting of the $v^{I_{-}}$coordinates then induces a splitting of the constrained $\operatorname{Spin}(7)$ moduli $\phi_{I_{-}}$defined in (3.30). After performing the redefinition (3.45) we may then move

\footnotetext{
${ }^{8}$ Recall that formally $\sigma\left(B_{3}\right)=-B_{3}$, since $\sigma$ reverses the orientation of $B_{3}$.

${ }^{9}$ They can be reduced by repeatedly using $\left(\omega^{0}\right)^{2}=-c_{1}\left(B_{3}\right) \wedge \omega_{0}$.
} 
into a set of redefined coordinates that are appropriate for performing the F-Theory lift. Firstly, $\phi^{0}$ is mapped the length of the interval and we set

$$
\frac{1}{r^{2}}=\phi^{0} \mathcal{V}^{-\frac{3}{4}},
$$

where $r$ is the circumference of the circle in $S^{1} / \mathbb{Z}_{2}$. Hence, $\phi^{0}$ captures degrees of freedom of the $4 \mathrm{~d}$ metric. The $\hat{\phi}^{\alpha-}$ become $4 \mathrm{~d}$ scalars, while the $\phi^{i}$ are the scalar part of $4 \mathrm{~d}$ vectors with index along the interval $\phi_{\mathrm{b}}^{i}=A_{3}^{i}$. It is convenient to set

$$
\phi_{\mathrm{b}}^{\alpha_{-}}=\left(\phi^{0}\right)^{\frac{1}{3}} \hat{\phi}^{\alpha_{-}}-\frac{1}{2}\left(\phi^{0}\right)^{-\frac{2}{3}} b^{\alpha} C_{i j} \phi^{i} \phi^{j}, \quad \mathcal{V}_{\mathrm{b}}=\left(\phi^{0}\right)^{\frac{1}{2}} \mathcal{V}^{\frac{9}{8}}, \quad \phi_{\mathrm{b}}^{i}=\left(\phi^{0}\right)^{-1} \phi^{i} .
$$

These redefinitions can be motivated by the fact that, when taking the F-theory limit with large $r$, the constraint (3.31) only depends on $\phi_{\mathrm{b}}^{\alpha_{-}}$, while $r$ and $\phi_{\mathrm{b}}^{i}$ are unconstrained. In addition, following [11] the vectors $A^{\alpha_{+}}$will become $4 \mathrm{~d}$ scalars with a real shift symmetry. We will consider the lift more explicitly in section 4.2 .

Let us finally also consider the flux-induced Chern-Simons couplings $\Theta_{I_{+} J_{+}}$and potential $F$, given in (3.35) and (3.36). From the split (3.42) we infer that the Chern-Simons coupling $\Theta_{\alpha_{+} \beta_{+}}$only involves vectors that become $4 \mathrm{~d}$ scalars and therefore, by the considerations of [39], have to be absent

$$
\Theta_{\alpha_{+} \beta_{+}}=0 \text {. }
$$

The real potential $F$ can be expressed in terms of $\Theta_{I_{-} I_{J}}$ as

$$
F=\int_{Y_{4}} G_{4} \wedge \operatorname{Re}(C \Omega)+\frac{1}{8} \mathcal{V}^{-1} \Theta_{I_{-} J_{-}} \phi^{I_{-}} \phi^{J_{-}} .
$$

Again using (3.42) and following [39] one has to additionally impose

$$
\Theta_{00}=0, \quad \Theta_{0 \alpha_{-}}=0, \quad \Theta_{0 i}=0, \quad \Theta_{\alpha_{-} \beta_{-}}=0, \quad \Theta_{i \beta_{-}}=0 .
$$

This choice of fluxes allows that a $4 \mathrm{~d}$ theory might exist, no fluxes are included in reduction from four to three dimensions, and the gauge-group $G$ is un-broken in four dimensions. ${ }^{10}$ The resulting potential $F$ will contain a term that is classical on the F-theory side and a one-loop contribution as we will discuss at the end of the next section.

\section{F-theory on $\operatorname{Spin}(7)$ manifolds}

In the previous section we studied M-theory on $\operatorname{Spin}(7)$ manifolds and later focused on examples constructed as quotients of elliptically fibered Calabi-Yau fourfolds by an antiholomorphic involution. As a next step we discuss in subsection 4.1 the dual interval reduction of a $4 \mathrm{~d}$ theory. Concretely, we will identify the boundary conditions on various $4 \mathrm{~d}$ fields on an interval that have to be imposed in order to make a duality of the form (1.1) possible. Aspects of the non-supersymmetric 4d effective theories are discussed in subsection 4.2. We particularly focus on the couplings of the uncharged scalar fields that are real both in three and four dimensions and satisfy Neumann boundary conditions at the ends of the interval.

\footnotetext{
${ }^{10}$ These conditions will be modified in the presence of $\mathrm{U}(1)$ gauge factors [36-38].
} 


\subsection{Dimensional reduction of the $4 d$ theory on an interval}

One of the crucial ingredients of the new kind of M-theory/F-theory duality claimed in (1.1) is the use of an interval in the dimensional reduction from four to three dimensions on the F-theory side of the duality. In this subsection we discuss some general features of dimensional reduction on an interval and consider candidate $4 \mathrm{~d}$ parent actions.

Due to the presence of an interval $I=S^{1} / \mathbb{Z}_{2}$ in (1.1) the up-lift of a $3 \mathrm{~d}$ theory on $\mathcal{M}_{3}$ to a $4 \mathrm{~d}$ theory on $\mathcal{M}_{4}=\mathcal{M}_{3} \times I$ is further complicated, since boundary conditions have to be given for each field. These have to be appropriately specified in order that the duality suggested in (1.1) holds. In the following we will discuss vectors, fermions, and scalars in turn.

Let us first consider a $4 \mathrm{~d}$ Abelian vector $A_{m}$. Since its components satisfy a secondorder equation of motion we can choose Dirichlet or Neumann conditions. This choice, however, has to be such that each component of the field strength $F_{m n}$ has a definite parity under the $\mathbb{Z}_{2}$ action. In particular, inspection of the the mixed component

$$
F_{\mu 3}=\partial_{\mu} A_{3}-\partial_{3} A_{\mu}
$$

reveals that if $A_{\mu}$ satisfies Dirichlet boundary conditions $A_{3}$ has to satisfy Neumann boundary conditions, and vice versa. This gives the two choices

$$
\begin{array}{llllll}
\text { (A) } D: & \left.A_{\mu}\right|_{\partial \mathcal{M}_{4}}=0 & \text { and } & N: & \left.\partial_{3} A_{3}\right|_{\partial \mathcal{M}_{4}}=0, \\
\text { (B) } & D: & \left.A_{3}\right|_{\partial \mathcal{M}_{4}}=0 & \text { and } & N: & \left.\partial_{3} A_{\mu}\right|_{\partial \mathcal{M}_{4}}=0,
\end{array}
$$

that may be made without over constraining the equation of motion. When carrying out the interval reduction the Dirichlet boundary conditions will remove the would-be zero mode of the corresponding $4 \mathrm{~d}$ field. So fields with Dirichlet boundary conditions will not be seen in the $3 \mathrm{~d}$ effective theory. This implies that reduction of $A_{m}$ can yield either one massless scalar or one massless vector in the $3 \mathrm{~d}$ effective action, but not both. This fact can be extended to non-Abelian gauge fields for a $4 \mathrm{~d}$ gauge group $G$. To do this let us denote the generators of the algebra of $G$ by $\left(T_{i}, T_{\mathcal{I}}\right)$, with $T_{i}$ labeling the Cartan generators. Then for each vector $A_{m}^{i}, A_{m}^{\mathcal{I}}$ one can choose different boundary conditions.

To conform with the theory arising in the $\operatorname{Spin}(7)$ reduction it turns out that one needs to chose option (A) in (4.2) for the Cartan vectors to keep $3 \mathrm{~d}$ scalars $\phi_{\mathrm{b}}^{i}=A_{3}^{i}$ and option (B) for the non-Cartan vectors in order to keep $3 \mathrm{~d}$ vectors $A_{\mu}^{\mathcal{I}} \cdot{ }^{11}$ In this case one notes that the non-Cartan $3 \mathrm{~d}$ vectors $A_{\mu}^{\mathcal{I}}$ acquire a mass term for which the mass is determined by the vacuum expectation value of the $3 \mathrm{~d}$ massless scalars $\phi_{\mathrm{b}}^{i}$. This mass term arises in the effective theory from the reduction of the gauge kinetic term. This analysis is consistent with the fact that the $3 \mathrm{~d}$ theory arising in the reduction described in section 3.3 is a Wilsonian effective action with no non-Cartan vectors and only the scalars $\phi^{i}, i=1, \ldots, \operatorname{rank}(G)$. Let us stress, however, that we are still able to extract the classical couplings using the $\operatorname{Spin}(7)$ reduction by uplifting the couplings of the scalars $\phi_{\mathrm{b}}^{i}$. The Lorentz transformations and gauge transformations of the $4 \mathrm{~d}$ vector mix all components

\footnotetext{
${ }^{11}$ These boundary conditions imply that the gauge coupling constant should be effectively assigned odd parity under the $\mathbb{Z}_{2}$ action.
} 
of $A_{m}^{i}, A_{m}^{\mathcal{I}}$ and thus allow to recover the couplings of the $4 \mathrm{~d}$ vectors from the couplings of $\phi_{\mathrm{b}}^{i}$, for a large interval on which these symmetries are restored.

Let us next consider a $4 \mathrm{~d}$ fermion given by a Majorana spinor $\chi$. Since its equations of motion are first-order, we can only impose a Dirichlet boundary condition of the form

$$
\left.\frac{1}{2}\left(1 \pm \gamma^{3}\right) \chi\right|_{\partial \mathcal{M}_{4}}=0
$$

without over constraining the dynamics. The sign is related to the intrinsic parity of the spinor under the $\mathbb{Z}_{2}$ action on the interval. For both choices, reduction of $\chi$ furnishes a massless Majorana spinor in the $3 \mathrm{~d}$ effective action. This implies that when focusing on zero modes, the degrees of freedom of the fermions are halved. However, there is no ambiguity when uplifting a fermion from three to four dimensions. 4d Lorentz invariance implies that the $3 \mathrm{~d}$ dynamics of the spinor encodes its $4 \mathrm{~d}$ couplings. A similar argument applies to the gravitino.

The comparison can, however, be more involved if the $4 \mathrm{~d}$ fermion is charged under the gauge group $G$. In an interval reduction the Coulomb branch scalars can give dimensionally reduced fermions a mass proportional to $\phi_{\mathrm{b}}^{i}$ if the coupling to $\phi_{\mathrm{b}}^{i}$ is non-vanishing. This implies that these fermions are not part of the low-energy effective theory and have to be integrated out. As with the vectors we find that the Cartan fermions remain dynamical in the $3 \mathrm{~d}$ low-energy effective theory. These then comprise the $3 \mathrm{~d}, \mathcal{N}=1$ supersymmetric partners of $\phi_{\mathrm{b}}^{i}$ moduli.

Finally, we turn to the reduction of a $4 \mathrm{~d}$ scalar field $\phi$ with standard two-derivative action yielding a second-order equation of motion. As a result, we can impose Dirichlet or Neumann boundary conditions

$$
\left.\phi\right|_{\partial \mathcal{M}_{4}}=0 \quad \text { or }\left.\quad \partial_{3} \phi\right|_{\partial \mathcal{M}_{4}}=0
$$

without over constraining the equation of motion. As a result the degree of freedom of a $4 \mathrm{~d}$ scalar might be entirely lost (for Dirichlet b.c.) or kept (for Neumann b.c.) when considering only the zero mode in the $3 \mathrm{~d}$ effective theory. This is in contrast to the vectors and fermions discussed above. In other words, one can add an arbitrary number of Dirichlet scalars to a candidate $4 \mathrm{~d}$ action without changing the $3 \mathrm{~d}$ effective theory on a small interval.

These features of interval reductions lead us to first specify a minimal 4d Lorentz invariant ansatz for the $4 \mathrm{~d}$ action containing only those couplings that can be uniquely fixed by comparison with the $3 \mathrm{~d} \mathcal{N}=1$ zero mode action. This non-supersymmetric theory is given to quadratic order in the fermions by

$$
\begin{aligned}
S_{\text {Min }}^{(4)}= & \int d^{4} x e\left[-\frac{1}{2} R-\frac{1}{2} \mathcal{G}_{\mathcal{A B}} \partial_{m} \varphi^{\mathcal{A}} \partial^{m} \varphi^{\mathcal{B}}-\frac{1}{4} f \operatorname{Tr}\left(F_{m n} F^{m n}\right)-V^{(4)}\right. \\
& -\frac{1}{2} \bar{\psi}_{m} \gamma^{m n r} D_{n} \psi_{r}-\frac{1}{2} \mathcal{G}_{\mathcal{A B}} \bar{\chi}^{\mathcal{A}} \gamma^{m} D_{m} \chi^{\mathcal{B}}-\frac{1}{2} f \operatorname{Tr}\left(\bar{\lambda} \gamma^{m} D_{m} \lambda\right)+\frac{1}{4} f \bar{\psi}_{m} \gamma^{r s} \gamma^{m} \operatorname{Tr}\left(\lambda F_{r s}\right) \\
& +\frac{1}{2 \sqrt{2}} \mathcal{G}_{\mathcal{A B}} \bar{\psi}_{m} \gamma^{n} \gamma^{m} \chi^{\mathcal{A}} D_{n} \varphi^{\mathcal{B}}+\frac{1}{2} A^{1} \bar{\psi}_{m} \gamma^{m n} \psi_{n}+\frac{1}{\sqrt{2}} A_{\mathcal{A}}^{2} \bar{\psi}_{m} \gamma^{m} \chi^{\mathcal{A}} \\
& \left.-\frac{1}{2} A_{\mathcal{A B}}^{3} \bar{\chi}^{\mathcal{A}} \chi^{\mathcal{B}}+\frac{1}{4 \sqrt{2}} A_{\mathcal{A}}^{4} \operatorname{Tr}\left(F_{m n} \bar{\lambda}\right) \gamma^{m n} \chi^{\mathcal{A}}-\frac{1}{2} \mathcal{G}^{\mathcal{A B}} A_{\mathcal{A}}^{4} A_{\mathcal{B}}^{2} \operatorname{Tr}(\bar{\lambda} \lambda)\right]
\end{aligned}
$$


where the covariant derivatives of the Majorana fermions are given by

$$
\begin{aligned}
& D_{m} \psi_{n}=\partial_{m} \psi_{n}+\frac{1}{4} \omega_{m r s} \gamma^{r s} \psi_{n}, \quad D_{m} \lambda=\partial_{m} \lambda+\frac{1}{4} \omega_{m r s} \gamma^{r s} \lambda+\left[A_{m}, \lambda\right] \\
& D_{m} \chi^{\mathcal{A}}=\partial_{m} \chi^{\mathcal{A}}+\frac{1}{4} \omega_{m r s} \gamma^{r s} \chi^{\mathcal{A}}+D_{m} \phi^{\mathcal{B}} \Gamma_{\mathcal{B C}}{ }^{\mathcal{A}} \chi^{\mathcal{C}}
\end{aligned}
$$

In this action $G_{\mathcal{A B}}$ is a real metric for the scalar target space and $V^{(4)}, f$ are real functions of the scalars $\varphi^{\mathcal{A}}$. In addition to this $A^{1}, A_{\mathcal{A}}^{2}, A_{\mathcal{A B}}^{3}$ and $A_{\mathcal{A}}^{4}$ are further functions of $\varphi^{\mathcal{A}}$ that will later be determined by comparing the reduction of this action with the $3 \mathrm{~d}$ result. As this action is not supersymmetric we could in principle have made a much more general proposal for the couplings that appear. However, it will turn out that (4.5) is sufficiently general to allow for a matching with the $3 \mathrm{~d}$ theory to be performed. For convenience we note here that performing this calculation one finds that the potential is given in terms of a real function $\mathcal{F}$ by

$$
V^{(4)}=2 G^{\mathcal{A B}} \partial_{\mathcal{A}} \mathcal{F} \partial_{\mathcal{B}} \mathcal{F}-3 \mathcal{F}^{2},
$$

and that the $A$ functions are given in terms of $\mathcal{F}$ and $f$ by

$$
A^{1}=\mathcal{F}, \quad A_{\mathcal{A}}^{2}=\partial_{\mathcal{A}} \mathcal{F}, \quad A_{\mathcal{A B}}^{3}=D_{\mathcal{A}} \partial_{\mathcal{B}} \mathcal{F}-\frac{1}{2} \mathcal{G}_{\mathcal{A B}} \mathcal{F} . \quad A_{\mathcal{A}}^{4}=\partial_{\mathcal{A}} f
$$

The action $S_{\text {Min }}^{(4)}$ given in (4.5) should be used with caution. It was constructed as the minimal functional consistent with $4 \mathrm{~d}$ Lorentz invariance that yields the $3 \mathrm{~d}$ action upon interval reduction. Note that this construction does not ensure conservation of the currents coupling to gravitini and gauge fields. This is needed in a consistent theory [40] and can be achieved by a suitable extension including supersymmetry [41]. Furthermore, we point out that the interpretation of (4.5) as a Wilsonian effective action is questionable, since it might not capture the dynamics of all light degrees of freedom. All scalars satisfying Dirichlet boundary conditions have only massive excitations for a finite interval lenght and are dropped from the action (4.5). For finite interval length these fields are strictly speaking not moduli even in the absence of fluxes. We will comment further on these Dirichlet scalars below, but will not discuss their impact in detail. They might, however, restore $4 \mathrm{~d}, \mathcal{N}=1$ supersymmetry in the $4 \mathrm{~d}$ bulk if the size of the interval is taken to infinity. ${ }^{12}$

A possible $4 \mathrm{~d}$ Wilsonian effective action $S_{\mathrm{W}}^{(4)}$ completing $S_{\text {Min }}^{(4)}$ on a large interval could be given by a $\mathcal{N}=1$ Lagrangian $\mathcal{L}_{\mathcal{N}=1}^{(4)}$ for F-theory on the original Calabi-Yau space $Y_{4}$ supplemented by the boundary conditions or a boundary action $\mathcal{L}^{(3)}$. Hence, it takes the form

$$
S_{\mathrm{W}}^{(4)}=\int_{\mathcal{M}_{4}} \mathcal{L}_{\mathcal{N}=1}^{(4)}+\int_{\partial \mathcal{M}_{4}} \mathcal{L}^{(3)}
$$

The restauration of the Calabi-Yau moduli space from the moduli space of the $\operatorname{Spin}(7)$ manifold in the large interval limit would be very non-trivial. In a follow-up paper we will explore this treatment further [23].

\footnotetext{
${ }^{12}$ We are grateful to Eran Palti and Ralph Blumenhagen for useful discussions on this point.
} 
Let us stress that the action (4.5) neglects the couplings of charged matter that will be present in a general F-theory compactification. Furthermore, we have not displayed the terms of higher order in the fermions. These can be added by making an Ansatz for these couplings and reducing them to three dimensions with the boundary conditions described above. The coefficients are then determined by comparing the zero mode result to a general $3 \mathrm{~d}, \mathcal{N}=1$ theory in which the higher fermionic couplings are known in terms of the $3 \mathrm{~d}$ $\mathcal{N}=1$ characteristic functions determined by the reduction of the terms in (4.5).

As stressed above the minimal action (4.5) could be modified by adding an arbitrary number of scalars satisfing Dirichlet boundary conditions without modifying the classical $3 \mathrm{~d}$ low-energy effective action for the zero modes. The question of determining the true 4d Wilsonian action can thus be not resolved from a purely supergravity perspective. A similar problem occurs for the ambiguities encountered in the up-lift of $3 \mathrm{~d} \mathcal{N}=2$ scalars on a circle in the standard M-theory F-theory duality. In such an up-lift a 3d scalar can either be part of a $4 \mathrm{~d} \mathcal{N}=1$ vector or chiral multiplet. The decisive information in determining this ambiguity arises form the M-theory to F-theory limit and the geometry of the Calabi-Yau fourfold. We will discuss this point further in what follows.

\subsection{Effective action of F-theory on $\operatorname{Spin}(7)$ manifolds}

Having described the $3 \mathrm{~d}$ effective theory obtained for the quotient torus fibered Spin(7) geometry in subsections 3.2 and 3.3 and the details on the interval reduction in subsection 4.1 we are now in the position to perform the reduction and read off the couplings of the $4 \mathrm{~d}$ theory (4.5). Clearly, proposing that the coupling functions take the same form in the $4 \mathrm{~d}$ theory is a speculative part of the analysis. It amounts on the one hand to sending the size of the interval $I$ to infinity, and on the other hand shrinking the fiber volume. This means that one has to be performing the M-theory to F-theory limit. In supersymmetric F-theory compactifications it has become clear over the last years $[11,36,42]$ that many couplings in the 3d theory obtained from M-theory appear to also have an F-theory interpretation. Motivated by these advances we perform a similar oxidation for the Spin(7) compactification. However, it should be stressed that we will only talk about zero modes in the following and many of the subtleties are, in fact, hidden in the treatment of massive modes.

The first step is to implement the F-theory limit explicitly. Note that not all couplings arising in the M-theory reduction are classical from the F-theory perspective on a small compact space. Various couplings can be induced at loop level when integrating out massive Coulomb branch and Kaluza-Klein modes. To extract the classical terms only, one can assert scalings to the various fields as suggested in [11]. The correct scalings are [42]

$$
v^{0} \rightarrow \epsilon v^{0}, \quad v^{\alpha_{-}} \rightarrow \epsilon^{-1 / 2} v^{\alpha_{-}}, \quad v^{i} \rightarrow \epsilon^{1 / 4} v^{i}, \quad r \rightarrow \epsilon^{-3 / 4} r .
$$

They ensure precisely that the couplings with intersection numbers (3.44), i.e. $\mathcal{K}_{0 \alpha_{-} \beta_{-}, \gamma_{-}}$, $\mathcal{K}_{0 \alpha_{-} \beta_{+}, \gamma_{+}}$and $\mathcal{K}_{i j \alpha_{-} \beta_{-}}, \mathcal{K}_{i j \alpha_{+} \beta_{+}}$are surviving the $\epsilon \rightarrow 0$ limit. Translated into the coordinates $\phi^{I_{-}}$one thus finds

$$
\phi^{0} \rightarrow \epsilon^{9 / 8} \phi^{0}, \quad \phi^{\alpha_{-}} \rightarrow \epsilon^{-3 / 8} \phi^{\alpha_{-}}, \quad \phi^{i} \rightarrow \epsilon^{3 / 8} \phi^{i}, \quad \mathcal{V} \rightarrow \epsilon^{-1 / 2} \mathcal{V}
$$


Combining these scalings with the coordinate redefinitions (3.47) one extracts the leading terms of all fields. We first introduce the $\phi_{\mathrm{b}}^{\alpha_{-}}$defined as the leading term in (3.47). In the limit the normalization constraint (3.31) translates to the condition

$$
N_{\mathrm{b}} \equiv \frac{1}{3 !} \kappa_{\alpha_{-} \beta_{-} \gamma_{-}} \phi_{\mathrm{b}}^{\alpha_{-}} \phi_{\mathrm{b}}^{\beta_{-}} \phi_{\mathrm{b}}^{\gamma_{-}}=1 \text {. }
$$

This implies that only $h_{-}^{1,1}\left(B_{3}\right)-1$ coordinates $\phi_{\mathrm{b}}^{\alpha_{-}}$are independent. The missing degree of freedom is encoded by the base volume $\mathcal{V}_{\mathrm{b}}$ arising as leading term in the definition (3.47). After the $\epsilon \rightarrow 0$ limit the resulting 3d action can be matched with a the reduction of a $4 \mathrm{~d}$ theory reduced on an interval of length $r$ with boundary conditions introduced in subsection 4.1. This allows us to read off the data of the $4 \mathrm{~d}$ theory from the $3 \mathrm{~d}$ action.

We first note that all couplings containing $3 \mathrm{~d}$ vectors or fermions are formally lifted from $3 \mathrm{~d}$ to $4 \mathrm{~d}$ in a Lorentz compatible way. For example, the kinetic terms in (3.1) for the $3 \mathrm{~d}$ fermions $\chi^{\alpha_{-}}$, which are in the same $3 \mathrm{~d}, \mathcal{N}=1$ multiplets as the scalars $\phi_{\mathrm{b}}^{\alpha_{-}}$, are given by

$$
\frac{1}{2} \tilde{g}_{\alpha_{-} \beta_{-}} \bar{\chi}^{\alpha_{-}} \not D \chi^{\beta_{-}}
$$

These are lifted by completing the $\chi^{\alpha_{-}}$into $4 \mathrm{~d}$ fermions and matching $\tilde{g}_{\alpha_{-} \beta_{-}}$with the reduction of the equivalent $4 \mathrm{~d}$ terms after performing the reduction and Weyl rescaling as well as implementing the $\epsilon \rightarrow 0$ limit with (4.11). In this way we can read off

$$
\mathcal{G}_{\alpha_{-} \beta_{-}}=\left(\tilde{g}_{\alpha_{-} \beta_{-}}\right)_{\epsilon=0}=4 \mathcal{V}_{\mathrm{b}}^{3} \int_{B_{3}} \xi_{\alpha_{-}}^{\mathrm{b}} \wedge * \xi_{\beta_{-}}^{\mathrm{b}},
$$

where the four-forms $\xi_{\alpha_{-}}^{\mathrm{b}}$ are given by

$$
\xi_{\alpha_{-}}^{\mathrm{b}}=\frac{1}{4} \mathcal{V}_{\mathrm{b}}^{-\frac{4}{3}} P_{\alpha_{-}}{ }^{\gamma_{-}} \omega_{\gamma_{-}} \wedge \omega_{\beta_{-}} \phi_{\mathrm{b}}^{\beta_{-}}, \quad P_{\alpha_{-}}^{\beta_{-}}=\delta_{\alpha_{-}}{ }^{\beta_{-}}-\frac{1}{3 !} \kappa_{\alpha_{-} \gamma_{-} \delta_{-}} \phi_{\mathrm{b}}^{\gamma_{-}} \phi_{\mathrm{b}}^{\delta_{-}} \phi_{\mathrm{b}}^{\beta_{-}} .
$$

The other components of the $4 \mathrm{~d}$ scalar metric $G_{\mathcal{A B}}$ appearing in (4.5) may then be deduced in a similar way by expanding $\varphi^{\mathcal{A}}=\left(\mathcal{V}_{b}, \phi^{\alpha_{-}}, \varphi^{\mathcal{K}}, \zeta_{\alpha_{+}}\right)$and making the comparison with (3.1) and (3.32). This gives $\mathcal{G}_{\mathcal{V}_{b}} \mathcal{V}_{b}=\frac{4}{6} \mathcal{V}_{\mathrm{b}}^{-2}$ and

$$
\mathcal{G}_{\mathcal{K} \mathcal{L}}=\left(\tilde{g}_{\mathcal{K} \mathcal{L}}\right)_{\epsilon=0}=4 \mathcal{V}_{\mathrm{b}}^{3} \int_{B_{3}} \xi_{\mathcal{K}}^{\mathrm{b}} \wedge * \xi_{\mathcal{L}}^{\mathrm{b}}, \quad \mathcal{G}^{\alpha_{+} \beta_{+}}=\left(h_{\alpha_{+} \beta_{+}}\right)_{\epsilon=0}^{-1}=\left(\frac{1}{2 \mathcal{V}_{b}} \int_{B_{3}} \omega_{\alpha_{+}} \wedge * \omega_{\beta_{+}}\right)^{-1}
$$

Next we can consider the comparison of the kinetic terms for the scalars $\phi^{i}$ with the reduction of the $4 \mathrm{~d}$ vector kinetic terms. In this way we find that the coupling function $f$ is given by

$$
f C_{i j}=\left(r^{2} g_{i j}\right)_{\epsilon=0}=\mathcal{V}_{\mathrm{b}}^{2 / 3} C_{i j} b_{S}^{\alpha_{-}} \kappa_{\alpha_{-} \beta_{-} \gamma_{-}} \phi_{\mathrm{b}}^{\beta_{-}} \phi_{\mathrm{b}}^{\gamma_{-}} .
$$

Similarly the reduction of the potential for the $4 \mathrm{~d}$ theory may be compared with the general $3 \mathrm{~d}, \mathcal{N}=1$ result (3.2) from which we find (4.7) where the function $\mathcal{F}$ is related to the function $F$, which determines the potential of the quotiented Calabi-Yau reduction, by

$$
\mathcal{F}=(r F)_{\epsilon=0}=\left(e^{K^{\mathrm{F}} / 2} \int_{Y_{4}} \operatorname{Re}(\Omega) \wedge G_{4}\right)_{\mathcal{L}_{\sigma}} .
$$


where $K^{\mathrm{F}}=-2 \log \mathcal{V}_{\mathrm{b}}-\log \int_{Y_{4}} \Omega \wedge \bar{\Omega}$. Finally we note that by comparing the fermionic couplings in the reduction of (4.5) with (3.1) we find (4.8).

We stress that in contrast to a supersymmetric effective theory the couplings of the bosons are less restricted and holomorphicity does neither protect the generating potential (4.18) nor the gauge coupling (4.17). It would be desirable to check if $3 \mathrm{~d}, \mathcal{N}=1$ supersymmetry helps to nevertheless ensures additional control over the corrections to these couplings on a finite size interval.

In the preceding analysis we did not include charged matter. Clearly, in a general Ftheory compactification with fluxes chiral matter will be part of the $4 \mathrm{~d}$ massless spectrum. This matter can become massive when dimensionally reduced on an interval if the scalars $\phi_{\mathrm{b}}^{i}$ get a vacuum expectation value. This implies that these have to be integrated out in the $3 \mathrm{~d}$ low-energy effective theory. In contrast to the $3 \mathrm{~d}, \mathcal{N}=2$ theories arising in Calabi-Yau fourfold compactifications [36-38] there is no one-loop contribution of chiral matter to $3 \mathrm{~d}$ Chern-Simons terms in our $3 \mathrm{~d}, \mathcal{N}=1$ setup. However, part of the $3 \mathrm{~d}$ potential $F$ will admit a one-loop term

$$
F \supset F^{\text {class }}+F^{1-\text { loop }} .
$$

This classical term will lift to the $4 \mathrm{~d}$ superpotential (4.18) in our simple configurations with only one unbroken non-Abelian gauge group. The one-loop term can be obtained by considering the general $\operatorname{Spin}(7)$ potential $\mathcal{F}$ with (3.36), imposing that up-lift conditions (3.50), and keeping the term that vanish in the limit $\epsilon \rightarrow 0$. This leads to the identification

$$
F^{1-\text { loop }} \stackrel{?}{=} \frac{1}{8} \mathcal{V}^{-2} \int_{Z_{4}} J \wedge J \wedge G_{4}=\frac{1}{8} \mathcal{V}^{-1} \Theta_{i j} \phi^{i} \phi^{j}
$$

It would be very interesting to check this match for an explicit example by computing both the general one-loop contribution in field theory and the flux intersection $\Theta_{i j}$ of the form (3.10).

Let us close with a brief comment on the Kaluza-Klein modes in the interval reduction. In the M-theory to F-theory duality on elliptically fibered Calabi-Yau manifolds the Kaluza-Klein modes map to M2-branes that wrap also the elliptic fiber. This implies that these states are charged under the Kaluza-Klein vector. In quotient torus fibered Spin(7) manifolds it is therefore crucial to investigate M2-brane states wrapping the fiber. Since, in the generic case of figure 3, the torus is mapped to an orientation reversed image, such M2-branes states appear in pairs. It remains to be checked which effects these have on the supersymmetry of the $3 \mathrm{~d}$ and $4 \mathrm{~d}$ effective theories. It is an important task to investigate these massive states to gain deeper insights into the correct choices of boundary conditions on the interval and the Kaluza-Klein compactification from $4 \mathrm{~d}$ to $3 \mathrm{~d}$.

\section{Comments on weak coupling and charged matter}

This section is devoted to the discussion of some aspects of the weak coupling limit for F-theory on $\operatorname{Spin}(7)$ manifolds. In particular, we focus on the case in which the $\operatorname{Spin}(7)$ manifold is a quotient torus fibration as described in section 2. We propose a Type IIB realization of the setup and we briefly comment on the charged matter spectrum in this string theory picture. 


\subsection{Weak coupling interpretation}

In what follows we describe a proposal for the Type IIB realization of the weak coupling limit of F-theory on Spin(7) manifolds constructed as anti-holomorphic quotients of CalabiYau fourfolds.

Before the anti-holomorphic involution is implemented we have F-theory on the fourfold $Y_{4}$ with base $B_{3}$. In the weak coupling limit [43] this becomes a Type IIB orientifold on a Calabi-Yau threefold $Y_{3}$ acted upon by a holomorphic involution $\sigma_{\text {hol }}$ in such a way that $B_{3}=Y_{3} / \sigma_{\text {hol }}$. After the implementation of the anti-holomorphic involution $\sigma$ on $Y_{4}$ on the M-theory side it is natural to expect a further quotient of the Type IIB setup under the associated involution $\sigma$ acting on the base $B_{3}$. In summary, we propose that the weak coupling picture of the setup under examination is furnished by Type IIB on the Calabi-Yau threefold $Y_{3}$ quotiented both by a holomorphic involution and by an antiholomorphic involution.

To make this proposal more precise we need to determine the intrinsic parities of Type IIB fields under the action of the anti-holomorphic involution. As in the standard discussion of the M-theory/F-theory duality [7] it is convenient to start with the simple case of M-theory on a product manifold $\mathcal{M}_{9} \times T^{2}$. The eleven-dimensional metric takes the form

$$
d s_{11}^{2}=\frac{v}{\tau_{2}}\left[\left(d x+\tau_{1} d y\right)^{2}+\tau_{2}^{2} d y^{2}\right]+d s_{9}^{2},
$$

where $x, y$ are coordinates one the torus, which has modular parameter $\tau=\tau_{1}+i \tau_{2}$. Mtheory is reduced on the circle parametrized by $x$ to get Type IIA. The $y$-circle is the T-duality circle. In this factorized case we consider an anti-holomorphic involution $\sigma$ that acts separately on $\mathcal{M}_{9}$ and $T^{2}$. More precisely, $\sigma$ acts on a complex three-dimensional submanifold $B_{3}$ of $\mathcal{M}_{9}$ as described in section 2.2. Equation (2.16) applied to the case of a constant fibration shows that $\tau$ has to be purely imaginary in order to have compatibility with the anti-holomorphic involution. As a result, the anti-holomorphic action $z \rightarrow \bar{z}$, where $z=x+\tau y$, is equivalent to $x \rightarrow x, y \rightarrow-y$.

The above observation is useful in establishing the intrinsic parities of Type IIB fields under the action of $\sigma$ by means of the following heuristic argument. To begin with, we associate negative intrinsic parity to the $y$ coordinate and positive intrinsic parity to the $x$ coordinate. Next, we observe that all M-theory fields have positive parity under $\sigma$. Finally, we use the standard chain of dualities to identify the M-theoretical origin of each Type IIB field and deduce its parity. ${ }^{13}$ This step is summarized in table 1 together with our findings for the intrinsic $\sigma$-parities.

In summary, we conjecture that the Type IIB weak coupling picture of the Spin(7) compactifications we are studying is obtained by taking the quotient under the symmetry group generated by the transformations

$$
\mathcal{O}_{\text {hol }}=(-)^{F_{L}} \Omega_{p} \sigma_{\text {hol }}, \quad \mathcal{O}=(-)^{F_{L}} P_{3} \hat{\sigma}
$$

\footnotetext{
${ }^{13}$ Our application of Buscher's rules is purely schematic and is intended only as a tool to read off the $\sigma$-parities of Type IIB fields.
} 


\begin{tabular}{|cccc|}
\hline IIB & IIA & M & $\sigma$-parity \\
\hline \hline$\phi$ & $\phi$ & $g_{x x}$ & + \\
\hline$g_{\mu \nu}$ & $g_{\mu \nu}$ & $g_{\mu \nu}$ & \\
$g_{\mu y}$ & $\left(B_{2}\right)_{\mu y}$ & $\left(C_{3}\right)_{\mu y x}$ & + \\
$g_{y y}$ & $g_{y y}$ & $g_{y y}$ & \\
\hline$\left(B_{2}\right)_{\mu \nu}$ & $\left(B_{2}\right)_{\mu \nu}$ & $\left(C_{3}\right)_{\mu \nu x}$ & - \\
$\left(B_{2}\right)_{\mu y}$ & $g_{\mu y}$ & $g_{\mu y}$ & \\
\hline$C_{0}$ & $\left(C_{1}\right)_{y}$ & $g_{x y}$ & - \\
\hline$\left(C_{2}\right)_{\mu \nu}$ & $\left(C_{3}\right)_{\mu \nu y}$ & $\left(C_{3}\right)_{\mu \nu y}$ & - \\
$\left(C_{2}\right)_{\mu y}$ & $\left(C_{1}\right)_{\mu}$ & $g_{\mu x}$ & \\
\hline$\left(C_{4}\right)_{\mu \nu \rho y}$ & $\left(C_{3}\right)_{\mu \nu \rho}$ & $\left(C_{3}\right)_{\mu \nu \rho}$ & - \\
\hline
\end{tabular}

Table 1. Schematic summary of type IIB fields with Type IIA duals, M-theory origin, and intrinsic $\sigma$-parity. Indices $\mu, \nu, \rho$ refer to the nine-dimensional manifold $\mathcal{M}_{9}, x$ refers to the direction of the M-theory circle, $y$ refers to the direction of the circle along which T-duality is performed.

The expression for $\mathcal{O}_{\text {hol }}$ is the familiar orientifold action, with left-moving space-time fermion number $F_{L}$ and world-sheet parity $\Omega_{p}$. The expression for $\mathcal{O}$ deserves some comments. Firstly, the inclusion of the factor $(-)^{F_{L}}$ is motivated by the intrinsic $\sigma$-parities of table 1. Secondly, we have decomposed the action of $\sigma$ into two involutions $\hat{\sigma}$ and $P_{3}$. On the one hand, the involution $\hat{\sigma}$ is the anti-holomorphic involution on $Y_{3}$ determined by the action of $\sigma$ on $B_{3}$. On the other hand, the involution $P_{3}$ is the reflection of one spatial direction in $\mathbb{R}^{1,3}$,

$$
P_{3}:\left(x^{0}, x^{1}, x^{2}, x^{3}\right) \rightarrow\left(x^{0}, x^{1}, x^{2},-x^{3}\right) .
$$

Without the factor of $P_{3}$ we would not have a symmetry of Type IIB. Indeed, the antiholomorphic involution $\hat{\sigma}$ induces a Pin-odd transformation of ten-dimensional spinors, which has to be counterbalanced by the Pin-odd action induced by $P_{3}$ to ensure compatibility with the definite chirality of fermions in Type IIB supergravity. Note that the inclusion of $P_{3}$ is consistent with the interpretation of $x^{3}$ as the coordinate that parametrizes the interval that decompactifies in the F-theory limit. A more detailed study of the $\mathcal{O}$ involution in the context of string theory on toroidal orientifolds is desirable and is left for future investigation. ${ }^{14}$

We conclude this section by analyzing the up-lift of the $3 \mathrm{~d}$ action for Kähler and complex structure moduli. This will establish a match of the $3 \mathrm{~d} \operatorname{Spin}(7)$ moduli with the Neumann scalars of a $4 \mathrm{~d}$ theory. Let us start with the Kähler moduli. In Type IIB language these are given by

$$
T_{\alpha}=\frac{1}{2 !} \int_{Y_{3}} \omega_{\alpha} \wedge J_{\mathrm{b}}^{2}+i \int_{Y_{3}} \omega_{\alpha} \wedge C_{4}
$$

\footnotetext{
${ }^{14}$ Note that the action of $\mathcal{O}$ is reminiscent of non-standard orbifold actions recently considered in [44].
} 
where now $\omega_{\alpha}$ and $J_{\mathrm{b}}$ are understood as $(1,1)$-forms on the double-cover $Y_{3}$ of the base $B_{3}$. Recall the split introduced before (3.41) of $H^{2}\left(B_{3}\right)$ that translates into a split of $H^{2}\left(Y_{3}\right)$ into positive and negative subspaces under the action of $\hat{\sigma}^{*}$. Note also that an expression of the form $\int_{Y_{3}} \lambda_{6}$ survives the $\hat{\sigma}$-projection only if $\lambda_{6}$ is negative under $\hat{\sigma}^{*}$. Using table 1 one finds that $C_{4}$ has negative parity under $P_{3} \hat{\sigma}$. This implies that the $4 \mathrm{~d}$ moduli after the $\mathcal{O}$ quotient should transform under $P_{3}$ as

$$
P_{3} \text {-even : } \operatorname{Re} T_{\alpha_{-}}, \operatorname{Im} T_{\alpha_{+}}, \quad P_{3} \text {-odd }: \operatorname{Re} T_{\alpha_{+}}, \operatorname{Im} T_{\alpha_{-}} \text {, }
$$

The $P_{3}$-even scalars match exactly with the $3 \mathrm{~d}$ moduli that survive the $\sigma$ quotient on the Calabi-Yau fourfold $Y_{4}$ on the M-theory side.

Let us now turn to complex structure moduli. From a Type IIB perspective, those correspond to complex structure moduli of the threefold $Y_{3}$, D7-brane moduli, and the axion-dilaton. The action of the anti-holomorphic involution $\hat{\sigma}$ on $Y_{3}$ is such that

$$
\hat{\sigma}^{*} \Omega^{3,0}=e^{2 i \theta} \overline{\Omega^{3,0}} .
$$

This is completely analogous to the corresponding $\sigma$-action on the fourfold $Y_{4}$. Imposing (5.6) one infers that the $P_{3}$-even complex structure moduli span a real subspace of the $4 \mathrm{~d} \mathcal{N}=1$ moduli space. With similar arguments it is possible to check the correspondence between $3 \mathrm{~d} \operatorname{Spin}(7)$ moduli and $4 \mathrm{~d} P_{3}$-even moduli related to D7-branes and the axion-dilaton.

It is important to highlight the generic presence of $P_{3}$-odd scalars. Such scalar degrees of freedom cannot have a constant non-vanishing profile along the $x^{3}$ direction, and therefore do not correspond to moduli in the $4 \mathrm{~d}$ theory. From a $4 \mathrm{~d}$ perspective on a finite interval such scalars arise only as massive excitations. In summary, we can state that the orientifold picture suggests that the $4 \mathrm{~d}$ moduli, which are $P_{3}$-even, are in one-to-one correspondence with the $\operatorname{Spin}(7)$ moduli in the $3 \mathrm{~d}$ action (3.32). The interpretation of the $P_{3}$-odd scalars from an M-theory perspective requires a better understanding of M2-brane states in our setup and seems not to be accessible within the context of $11 \mathrm{~d}$ supergravity.

\subsection{Aspects of charged matter}

The effective action derived in the previous sections does not furnish an explicit description of the charged matter spectrum of F-theory on the class of Spin(7) manifolds under consideration. Charged matter becomes massive after the gauge group is broken to the Coulomb branch and is integrated out.

To get information about charged matter we can alternatively start looking at the weak coupling limit of our F-theory setup, discussed in the previous section. It can contain D7-branes that wrap holomorphic cycles in the threefold $Y_{3}$ and have $(1,1)$-type worldvolume flux to ensure the presence of $4 \mathrm{~d}$ chiral fermions. As we have seen, the crucial new ingredient is the anti-holomorphic involution $\hat{\sigma}$ combined with the transformation $P_{3}$ to have a symmetry of Type IIB.

We can specialize further and consider a point in moduli space in which the CalabiYau threefold $Y_{3}$ is realized as a toroidal orbifold. In this toroidal setups the embedding 
of D7-branes is described by one linear holomorphic equation for the flat complex coordinates of the torus. Information about the charged matter spectrum can be obtained by first principles, by quantizing open strings stretching between D7-branes. We can make some general remarks on the interplay between holomorphically embedded D7-branes and the anti-holomorphic involution. First of all, the image branes are also holomorphically embedded, if the anti-holomorphic action is linear in the flat coordinates of the torus. Second of all, the world-volume flux of an image brane is still of (1,1)-type, but its sign is reversed compared to the original brane. These considerations imply that if we start with a supersymmetric setup that contains only holomorphic branes with $(1,1)$ fluxes, these features are not spoiled by the introduction of image branes under the anti-holomorphic involution. Any intersection of any two branes or image branes possesses at least one complex massless scalar. Of course, one has to take into account the projection onto invariant states to determine if supersymmetry is actually present, or if different number of bosonic and fermionic massless states is projected out.

It is possible to argue that the robust features of the charged matter spectrum are insensitive to the details of the full compactification setup, and only depend on the local geometry around the intersection of the two D7-branes. This can be effectively described by looking at a non-compact model with flat D7-branes in $\mathbb{R}^{1,3} \times \mathbb{C}^{3}$. It captures the neighborhood of a fixed locus on the base $B_{3}$. Therefore the anti-holomorphic action $\sigma$ in local coordinates can be taken to be one of the maps given in (2.11). If $\hat{\sigma}$ does not square to the identity, its square is included as an additional holomorphic orbifold action, in such a way that $\hat{\sigma}^{2}=\mathbb{1}$ in the quotient space. We have performed explicitly the projection onto invariant states for the two linear actions in (2.11), and we have compared the result with the purely orientifold projection without the anti-holomorphic involution $\hat{\sigma}$ and without $P_{3}$. We have found that in both cases the same number of bosonic and fermionic degrees of freedom survives the projection. This signals that the charged matter spectrum is $\mathcal{N}=1$ supersymmetric also after the anti-holomorphic orbifold action is taken into account.

It can be checked that, irrespectively of the position of the D7-branes and their images under the action of $\hat{\sigma}$, no open string state can be invariant under the action of $\hat{\sigma} P_{3}$, but rather that open string states are always swapped in pairs. This seems to prevent an undemocratic truncation of the spectrum in such a way that the same number of bosonic and fermionic degrees of freedom is obtained. This general feature can be related to a mismatch between holomorphic embedding and anti-holomorphic involution. On the one hand, charged matter is localized at the intersection of two D7-branes, which is a complex one-dimensional holomorphic subspace of the internal six-torus. On the other hand, the fixed locus of the anti-holomorphic involution is either a real one-dimensional subspace (see the first action in (2.11)), or a real three-dimensional subspace incompatible with the holomorphic structure (see the second action in (2.11)). It is therefore impossible to have the intersection inside the fixed locus of the anti-holomorphic involution.

There are many other interesting open questions that can be addressed in toroidal models. For instance, it might be possible to relate closed string twisted sectors of the anti-holomorphic orbifold action to resolution modes of the Spin(7) geometry. We leave these investigations for future research. 


\section{Conclusions}

In this paper we studied aspects of $4 d$ effective theories arising from F-theory on $\operatorname{Spin}(7)$ manifolds. To approach this problem we proposed the duality (1.1) between an M-theory compactification on a certain fibered Spin(7) geometry and F-theory on a resolved version of this geometry multiplied by an interval, in the shrinking fiber limit. This provides the opportunity to study $4 \mathrm{~d}$ theories from F-theory by using $3 \mathrm{~d}$ minimally supersymmetric theories. We argued that these $\operatorname{Spin}(7)$ compactifications of F-theory can be approached via M-theory, when definite boundary conditions for the various $4 \mathrm{~d}$ fields on the interval are chosen. Our analysis focused on the comparison of the $3 \mathrm{~d}, \mathcal{N}=1$ zero mode actions on the M-theory and F-theory side. Up-lifting to four dimensions is the most conjectural step. However, making an appropriate minimal ansatz for the $4 \mathrm{~d}$ theory (4.5) on a finite interval, its couplings and general features can be determined compatible with the $\operatorname{Spin}(7)$ reduction of M-theory. In particular one identifies the $4 \mathrm{~d}$ scalar potential (4.7) and fermionic couplings (4.8). The study of the complete $4 \mathrm{~d}$ Wilsonian effective action is complicated by the fact that in the F-theory limit M2-brane states will become light and can introduce new $4 \mathrm{~d}$ degrees of freedom. These can be light in the limit of a large interval and might help to restore $4 \mathrm{~d}, \mathcal{N}=1$ supersymmetry away from the interval boundaries. This would be in the same spirit as [45].

To provide evidence for the M-theory to F-theory duality it was crucial to specify a class of $\operatorname{Spin}(7)$ manifolds for which this duality can be analyzed. Concretely, we employed Spin(7) manifolds that are obtained by quotienting an elliptically fibered Calabi-Yau fourfold by an anti-holomorphic and isometric involution $\sigma$. We called the resulting manifolds quotient torus fibered Spin(7) manifolds. Consequently, the 3d effective theories arising when compactifying M-theory on these quotient torus fibrations respect only the minimal number $(\mathcal{N}=1)$ of supersymmetries. These can be obtained by truncating the $\mathcal{N}=2$ theory arising in the Calabi-Yau fourfold reduction of M-theory. More generally, we have revisited the $3 \mathrm{~d}, \mathcal{N}=1$ effective theories arising in M-theory compactifications on smooth Spin(7) manifolds with four-form fluxes. We determined the characteristic data of the zero mode theories in terms of the internal geometry. The $3 \mathrm{~d}$ theories obtained from the quotiented Calabi-Yau spaces were shown to comprise a special class of such $3 \mathrm{~d}, \mathcal{N}=1$ theories. The same class of theories was then shown to arise from an interval reduction of specific $4 \mathrm{~d}$ non-supersymmetric theories if appropriate boundary conditions on the $4 \mathrm{~d}$ fields are imposed.

The spectrum and couplings of the $4 \mathrm{~d}$ theories were constrained to yield a $3 \mathrm{~d}$ zero mode action that matches with the Spin(7) reduction. This imposes stringent constraints on the allowed $4 \mathrm{~d}$ theories. We have argued that $4 \mathrm{~d}$ vectors can still be grouped with fermions similar to $4 \mathrm{~d}, \mathcal{N}=1$ vector multiplets. The classical couplings of the vector fields and their fermionic partners are determined by $3 \mathrm{~d}$ couplings of the $3 \mathrm{~d}, \mathcal{N}=1$ Coulomb branch scalars $\phi^{i}$ by Lorentz symmetry and gauge symmetry. These symmetries should be restored in the interior of a very large interval. Similarly, one can proceed with the couplings of the $4 \mathrm{~d}$ metric and gravitino that are constrained by the $3 \mathrm{~d}$ couplings and $4 \mathrm{~d}$ Lorentz symmetry. It should be stressed, however, that the $4 \mathrm{~d}$ couplings are no longer 
supersymmetric in the minimal action (4.5) that can be unambiguously determined from the $3 \mathrm{~d}$ M-theory reduction. This is due to the fact that one of the two real scalars in a $4 \mathrm{~d} \mathcal{N}=1$ chiral multiplet would need to arise in the M-theory to F-theory limit from M2-brane states. In fact, we argued that one can map the Spin(7) moduli to only one of the real scalars in these multiplets. The kinetic terms of the real moduli scalars and the form of the scalar potential were discussed in section 4.2. They are less constrained than in $4 \mathrm{~d}, \mathcal{N}=1$ theories, but still inherit special properties from the class of $\operatorname{Spin}(7)$ geometries used in our work. Since for our construction there is always an underlying Calabi-Yau fourfold, $4 \mathrm{~d} \mathcal{N}=1$ supersymmetry might be locally present away from the boundaries. To complete this picture it would be desirable to study Kaluza-Klein modes arising in the interval compactification and obtaining a $3 \mathrm{~d}$ action including the dynamics of all such modes. ${ }^{15}$

In should be noted that the constructions of $\operatorname{Spin}(7)$ manifolds originally proposed in [12] also included isolated orbifold points coinciding with the fixed points of $\sigma$. Showing that these can be resolved in a Spin(7)-compatible fashion was a crucial task in [12]. We have not included a study of these modes in this work, but it would be very interesting to understand how they modify the $4 \mathrm{~d}$ effective theory. In particular, we found that if $\sigma$ has only isolated fixed points on $Y_{4}$ that the torus must be pinched over these points. This suggests an interesting link between the gauge theory dynamics and the singularities that need to be resolved in a Spin(7)-compatible way to obtain a smooth geometry. As for ordinary non-Abelian gauge theory singularities of elliptically fibered Calabi-Yau fourfolds, F-theory might be well-defined on the singular Spin(7) geometry if one can identify the new light states arising near the singularities.

A complete understanding of the supersymmetry breaking in our proposed approach will require a more detailed understanding of the $4 \mathrm{~d}$ Wilsonian effective action. An important role in the supersymmetry breaking is played by the presence of the boundaries. If one assumes a $4 \mathrm{~d}$ Wilsonian action of the form (4.9) the scale at which the $4 \mathrm{~d} \mathcal{N}=1$ supersymmetry is broken is related to the size of the inteval.

A further interesting open problem is to understand which corrections the $4 \mathrm{~d}$ action of scalars in our reduction will admit. In particular, the scalar fields $\phi_{\mathrm{b}}^{\alpha}$ and the volume $\mathcal{V}_{\mathrm{b}}$ of the base $B_{3}$ are massless in the $\operatorname{Spin}(7)$ holonomy reductions presented here and it would be interesting to see which effects render these fields massive as, similarly to CalabiYau fourfold compactifications, $G_{4}$ fluxes cannot stabilize all Kähler moduli. We should, however, stress that we used fluxes only without including their back-reaction and it would be interesting to consider reductions on the back-reacted backgrounds of [17, 28, 29].

Let us close by noting that many aspects of our proposal have only been addressed very briefly despite their imminent importance. We have only briefly discussed the weak coupling interpretation and the charged matter spectrum. The quantization of Type IIB string theory on the constructed backgrounds is an interesting open task to which we hope to return in the near future. This should also shed more light on the string interpretation of the singularities induced in the $\operatorname{Spin}(7)$ construction and the presence of an interval.

\footnotetext{
${ }^{15}$ For actions of this type in a circle reduction, see, for example, [46, 47].
} 
The crucial observation has been that a simple circle reduction cannot connect the $4 \mathrm{~d}$ and 3d effective theories of F-theory and M-theory. This might admit alternative realizations, for example in Sherck-Schwarz reductions, which provide exciting further directions to implement such dualities.

\section{Acknowledgments}

We would like to thank Carlo Angelantonj, Jan Keitel, Dieter Lüst, Wati Taylor, Paul Townsend, and Hagen Triendl for interesting discussions and correspondence. We are particularly grateful to Eran Palti and Ralph Blumenhagen for their useful comments on the first version of this work. T.G. would like to thank the High Energy group of Harvard University for its hospitality. This work was supported by a research grant of the Max Planck Society.

\section{A Conventions}

For every space-time dimension $d$ we choose the mostly plus signature for the metric $g_{\mu \nu}$ and we adopt the following conventions for the Riemann tensor:

$$
\begin{aligned}
\Gamma_{\mu \nu}^{\rho} & =\frac{1}{2} g^{\rho \sigma}\left(\partial_{\mu} g_{\nu \sigma}+\partial_{\nu} g_{\mu \sigma}-\partial_{\sigma} g_{\mu \nu}\right) \\
R_{\tau \mu \nu}^{\lambda} & =\partial_{\mu} \Gamma_{\nu \tau}^{\lambda}-\partial_{\nu} \Gamma_{\mu \tau}^{\lambda}+\Gamma_{\mu \alpha}^{\lambda} \Gamma_{\nu \tau}^{\alpha}-\Gamma_{\nu \alpha}^{\lambda} \Gamma_{\mu \tau}^{\alpha}, \quad R_{\mu \nu}=R_{\mu \lambda \nu}^{\lambda}, \quad R=R_{\mu \nu} g^{\mu \nu} .
\end{aligned}
$$

The Levi-Civita tensor is denoted by $\epsilon_{\mu_{1} \ldots \mu_{d}}$. In our conventions it satisfies

$$
\epsilon_{01 \ldots(d-1)}=\sqrt{-\operatorname{det} g_{\mu \nu}}
$$

in any coordinate system $\left(x^{0}, x^{1}, \ldots, x^{d-1}\right)$. Differential $p$-forms are expanded on the basis of differential of the coordinates as

$$
\lambda=\frac{1}{p !} \lambda_{\mu_{1} \ldots \mu_{p}} d x^{\mu_{1}} \wedge \cdots \wedge d x^{\mu_{p}}
$$

so that the wedge product of a $p$ - and a $q$-form satisfies

$$
(\alpha \wedge \beta)_{\mu_{1} \ldots \mu_{p+q}}=\frac{(p+q) !}{p ! q !} \alpha_{\left[\mu_{1} \ldots \mu_{p}\right.} \beta_{\left.\mu_{p+1} \ldots \mu_{p+q}\right]} .
$$

Exterior differentiation of a $p$-form is given by

$$
(d \alpha)_{\mu_{0} \ldots \mu_{p}}=(p+1) \partial_{\left[\mu_{0}\right.} \alpha_{\left.\mu_{1} \ldots \mu_{p}\right]} .
$$

The Hodge dual of $p$-form in real coordinates and arbitrary space-time dimension $d$ is defined by the expression

$$
(* \alpha)_{\mu_{1} \ldots \mu_{d-p}}=\frac{1}{p !} \alpha^{\nu_{1} \ldots \nu_{p}} \epsilon_{\nu_{1} \ldots \nu_{p} \mu_{1} \ldots \mu_{d-p}},
$$

in such a way that

$$
\alpha \wedge * \beta=\frac{1}{p !} \alpha_{\mu_{1} \ldots \mu_{p}} \beta^{\mu_{1} \ldots \mu_{p}} * 1
$$

holds for arbitrary $p$-forms $\alpha, \beta$. 


\begin{tabular}{|c|c|c|c|c|}
\hline \multicolumn{2}{|r|}{ vertices } & coords. & $Q_{1}$ & $Q_{2}$ \\
\hline$\nu_{1}=(1$ & $\left.\begin{array}{llll}0, & 0, & 0, & 0\end{array}\right)$ & $x$ & 8 & 2 \\
\hline$\nu_{2}=(\quad 0$ & $\left.\begin{array}{llll}1, & 0, & 0, & 0\end{array}\right)$ & $y$ & 12 & 3 \\
\hline$\nu_{3}=(-2$ & $\left.\begin{array}{llll}-3, & 0, & 0, & 0\end{array}\right)$ & $z$ & 0 & 1 \\
\hline$\nu_{4}=(-2$ & $-3,-1,-1,-1)$ & $u_{1}$ & 1 & 0 \\
\hline$\nu_{5}=(-2$ & $\left.\begin{array}{llll}-3, & 1, & 0, & 0\end{array}\right)$ & $u_{2}$ & 1 & 0 \\
\hline$\nu_{6}=(-2$ & -3 & $u_{3}$ & 1 & 0 \\
\hline$\nu_{7}=(-2$ & -3 & $u_{4}$ & 1 & 0 \\
\hline
\end{tabular}

Table 2. Toric data for a reflexive polyhedron describing a $\mathbb{P}_{2,3,1}$ fibration of $\mathbb{P}_{1,1,1,1}$.

\section{B Example Spin(7) holonomy manifolds}

\section{B.1 A hypersurface in a $\mathbb{P}_{2,3,1}$ fibration of $\mathbb{P}_{1,1,1,1}$}

Let us consider a simple example of the construction described in section 2 in which the Calabi-Yau fourfold $Y_{4}$ is described by a polynomial in a toric ambient space constructed by fibering the weighted projective space $\mathbb{P}_{2,3,1}$ over $\mathbb{P}_{1,1,1,1}$. In the language of toric geometry this is described by a reflexive polyhedron with the set of rays given in table 2 .

This gives a smooth ambient space in which the Calabi-Yau fourfold will be defined by a homogeneous degree $(24,6)$ polynomial in the $\left(Q_{1}, Q_{2}\right)$ identifications. This polynomial may be brought into the Weierstrass form (2.9) where now the coefficients $f$ and $g$ are degree 16 and 24 , homogeneous polynomials of the base coordinates $u_{1}, \ldots, u_{4}$, respectively. A sufficiently general set of coefficients for these polynomials will then give a smooth CalabiYau fourfold. Next we impose a symmetry of this space under the action of the antiholomorphic involution $\sigma$ where

$$
\sigma\left(u_{1}, u_{2}, u_{3}, u_{4}, x, y, z\right)=\left(\bar{u}_{2},-\bar{u}_{1}, \bar{u}_{4},-\bar{u}_{3}, \bar{x}, \bar{y}, \bar{z}\right)
$$

This restricts the coefficients of the polynomial. However these coefficients remain general enough that a generic polynomial is still non-singular. The identification $\sigma$ has no fixed space on the base, as the would-be fixed space $u_{1}=u_{2}=u_{3}=u_{4}=0$ is removed by the Stanley-Reisner ideal. Every point of the base then represents an example of situation (1) as described in section 2 and so the $\operatorname{Spin}(7)$ holonomy manifold ${ }^{16}$ produced upon quotienting by $\sigma$ is non-singular. This means that no additional resolutions need to be performed.

\section{B.2 A complete intersection in a $\mathbb{P}_{1,1,1,1}$ fibration of $\mathbb{P}_{1,1,2,2}$}

Next let us consider a second construction in which the ambient space is formed by fibering $\mathbb{P}_{1,1,1,1}$ over $\mathbb{P}_{1,1,2,2}$. In this case the Calabi-Yau is given by a complete intersection of two polynomials described the following nef-partition in table 3 .

The two polynomials $P_{1}$ and $P_{2}$ are then associated with the partitions $\nabla_{1}$ and $\nabla_{2}$ respectively. These are both degree $(4,2)$ under identifications $\left(Q_{1}, Q_{2}\right)$.

\footnotetext{
${ }^{16}$ Note that strictly speaking the quotient manifold is expected to have $\mathrm{SU}(4) \times \mathbb{Z}_{2}$ holonomy.
} 


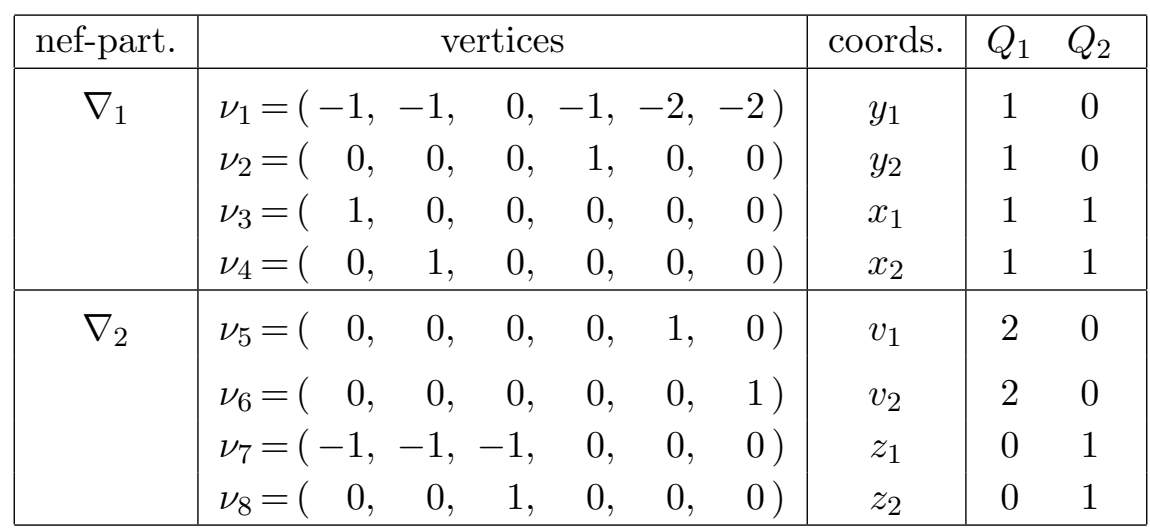

Table 3. Toric data for a nef-partition describing a C.I. in a $\mathbb{P}_{1,1,1,1}$ fibration of $\mathbb{P}_{1,1,2,2}$.

In this case the base $\mathbb{P}_{1,1,2,2}$ has a complex one-dimensional holomorphic orbifold singularity at $y_{1}=y_{2}=0$ before considering any anti-holomorphic quotient. This lifts to two separate complex two-dimensional singular spaces in the total ambient space. One, which is associated with the $Q_{1}$ identification, lies at $y_{1}=y_{2}=x_{1}=x_{2}=0$ and the other, which is associated with the $Q_{1}-Q_{2}$ identification, lies at $y_{1}=y_{2}=z_{1}=z_{2}=0$.

Let us first consider the singular space which lies at $y_{1}=y_{2}=x_{1}=x_{2}=0$. At this locus the polynomials can be written as

$$
P_{1}=a_{1} z_{1}^{2}+b_{1} z_{1} z_{2}+c_{1} z_{2}^{2} \quad P_{2}=a_{2} z_{1}^{2}+b_{2} z_{1} z_{2}+c_{2} z_{2}^{2}
$$

where $a_{1,2} b_{1,2}$ and $c_{1,2}$ are homogeneous quadratics in $v_{1}$ and $v_{2}$. The singularities of the ambient space will then intersect both polynomials at the places where one of the roots of $P_{1}$ sits on top of one of the roots of $P_{2}$. At these points the resultant of the pair of polynomials, given by

$$
-a_{2} b_{1} b_{2} c_{1}+a_{1} b_{2}^{2} c_{1}+a_{2}^{2} c_{1}^{2}+a_{2} b_{1}^{2} c_{2}-a_{1} b_{1} b_{2} c_{2}-2 a_{1} a_{2} c_{1} c_{2}+a_{1}^{2} c_{2}^{2},
$$

will vanish. This resultant is a homogeneous octic in $v_{1,2}$ so gives eight $\mathbb{Z}_{2}$ singular points on the Calabi-Yau fourfold at which the pair of the polynomials hit the two-dimensional space of singularities in the ambient space.

Next let us consider the singular space which lies at $y_{1}=y_{2}=z_{1}=z_{2}=0$. As before both polynomials will intersect the singularity of the ambient space when the resultant vanishes. This second resultant is a homogeneous quartic in $v_{1,2}$ so gives four $\mathbb{Z}_{2}$ singular points.

The Calabi-Yau fourfold may have extra singularities associated with the pinching of the torus. To find out where this happens we may make use of the singularity classification described in [48]. This shows that for a generic set of polynomial coefficients the torus pinches with a Type $I_{1}$ singularity over the intersection of a homogeneous degree $(72,0)$ polynomial in the $\left(Q_{1}, Q_{2}\right)$ identification, with the two polynomials that define the Calabi-Yau. Furthermore we find that this space intersects each of the $\mathbb{Z}_{2}$ singular points described above. 
We now impose a symmetry under the action of the anti-holomorphic involution $\sigma$ defined by,

$$
\sigma\left(y_{1}, y_{2}, v_{1}, v_{2}, x_{1}, x_{2}, z_{1}, z_{2}\right)=\left(\bar{y}_{2},-\bar{y}_{1}, \bar{v}_{2}, \bar{v}_{1}, \bar{x}_{2},-\bar{x}_{1}, \bar{z}_{2}, \bar{z}_{1}\right)
$$

As before this constrains the coefficients of the polynomials but does not alter the singularity structure of the Calabi-Yau. We note also that in this case $\sigma$ is not an involution on its own but that the identification $Q_{1}$ must be used to make $\sigma^{2}=\mathbb{1}$.

The action of $\sigma$ on the base gives a real one-dimensional fixed line which sits inside the holomorphic orbifold singularity of $\mathbb{P}_{1,1,2,2}$. At most places over this fixed line the torus is unpinched and has no fixed space. It represents an example of situation (2.1) described in section 2. However when the torus pinches over the fixed line of the base the pinched point on the torus becomes fixed under the action of $\sigma$ and so represents an example of situation (3). In additional, these fixed pinched points on the torus also lie at the eight $\mathbb{Z}_{2}$ singular points at $y_{1}=y_{2}=x_{1}=x_{2}=0$. By comparison the four $\mathbb{Z}_{2}$ singular points, which lie at $y_{1}=y_{2}=z_{1}=z_{2}=0$ are not fixed under $\sigma$ but instead are mapped pairwise into each other.

The quotient of this Calabi-Yau by $\sigma$ then gives a singular $\operatorname{Spin}(7)$ manifold. The presence of these singularities is not a problem in F-theory as this is defined on singular spaces. However in order to use the M-theory duality we have described to find the effective action these singularities must be resolved in an appropriate fashion. It is unclear how one would carry out this resolution or even if such a resolution can be performed at all for this particular Spin(7) manifold. For this reason it will be extremely important to investigate these resolutions further in future work.

Open Access. This article is distributed under the terms of the Creative Commons Attribution License (CC-BY 4.0), which permits any use, distribution and reproduction in any medium, provided the original author(s) and source are credited.

\section{References}

[1] R. Blumenhagen, M. Cvetič, P. Langacker and G. Shiu, Toward realistic intersecting D-brane models, Ann. Rev. Nucl. Part. Sci. 55 (2005) 71 [hep-th/0502005] [InSPIRE].

[2] R. Blumenhagen, B. Körs, D. Lüst and S. Stieberger, Four-dimensional String Compactifications with D-branes, Orientifolds and Fluxes, Phys. Rept. 445 (2007) 1 [hep-th/0610327] [INSPIRE].

[3] H.P. Nilles, S. Ramos-Sanchez, M. Ratz and P.K. Vaudrevange, From strings to the MSSM, Eur. Phys. J. C 59 (2009) 249 [arXiv:0806.3905] [inSPIRE].

[4] T. Weigand, Lectures on F-theory compactifications and model building, Class. Quant. Grav. 27 (2010) 214004 [arXiv: 1009.3497] [INSPIRE].

[5] A. Maharana and E. Palti, Models of Particle Physics from Type IIB String Theory and F-theory: A Review, Int. J. Mod. Phys. A 28 (2013) 1330005 [arXiv:1212.0555] [inSPIRE].

[6] C. Vafa, Evidence for F-theory, Nucl. Phys. B 469 (1996) 403 [hep-th/9602022] [InSPIRE].

[7] F. Denef, Les Houches Lectures on Constructing String Vacua, arXiv:0803.1194 [INSPIRE]. 
[8] M. Berger, Sur les groupes d'holonomie homogène des variétés riemanniennes, Bull. Soc. Math. France 83 (1955) 279.

[9] E. Witten, Is supersymmetry really broken?, Int. J. Mod. Phys. A 10 (1995) 1247 [hep-th/9409111] [INSPIRE].

[10] E. Witten, Strong coupling and the cosmological constant, Mod. Phys. Lett. A 10 (1995) 2153 [hep-th/9506101] [INSPIRE].

[11] T.W. Grimm, The $N=1$ effective action of F-theory compactifications, Nucl. Phys. B 845 (2011) 48 [arXiv: 1008.4133] [inSPIRE].

[12] D. Joyce, A new construction of compact 8 manifolds with holonomy spin(7), J. Diff. Geom. 53 (1999) 89 [math/9910002] [INSPIRE].

[13] M. Cvetič, G. Gibbons, H. Lü and C. Pope, New complete noncompact spin(7) manifolds, Nucl. Phys. B 620 (2002) 29 [hep-th/0103155] [inSPIRE].

[14] M. Cvetič, G. Gibbons, H. Lü and C. Pope, Cohomogeneity one manifolds of spin(7) and $G_{2}$ holonomy, Phys. Rev. D 65 (2002) 106004 [hep-th/0108245] [INSPIRE].

[15] E. Cremmer, B. Julia and J. Scherk, Supergravity Theory in Eleven-Dimensions, Phys. Lett. B 76 (1978) 409 [INSPIRE].

[16] G. Papadopoulos and P. Townsend, Compactification of $D=11$ supergravity on spaces of exceptional holonomy, Phys. Lett. B 357 (1995) 300 [hep-th/9506150] [INSPIRE].

[17] K. Becker, Anote on compactifications on spin(7)-holonomy manifolds, JHEP 05 (2001) 003 [hep-th/0011114] [INSPIRE].

[18] S. Gukov and J. Sparks, M theory on spin(7) manifolds. 1, Nucl. Phys. B 625 (2002) 3 [hep-th/0109025] [INSPIRE].

[19] G. Curio, B. Körs and D. Lüst, Fluxes and branes in type-II vacua and M-theory geometry with $G_{2}$ and spin(7) holonomy, Nucl. Phys. B 636 (2002) 197 [hep-th/0111165] [INSPIRE].

[20] B.S. Acharya, X. de la Ossa and S. Gukov, G flux, supersymmetry and spin(7) manifolds, JHEP 09 (2002) 047 [hep-th/0201227] [INSPIRE].

[21] S. Gukov, J. Sparks and D. Tong, Conifold transitions and five-brane condensation in M-theory on spin(7) manifolds, Class. Quant. Grav. 20 (2003) 665 [hep-th/0207244] [INSPIRE].

[22] M. Becker et al., $M$ theory on spin(7) manifolds, fluxes and 3-D, $N=1$ supergravity, Nucl. Phys. B 683 (2004) 67 [hep-th/0312040] [INSPIRE].

[23] F. Bonetti, T.W. Grimm, E. Palti and T.G. Pugh, F-Theory on Spin(7) Manifolds: Weak-Coupling Limit, arXiv:1309.2287 [INSPIRE].

[24] D.D. Joyce, Compact manifolds with special holonomy, Oxford University Press, Oxford U.K. (2000).

[25] R. Blumenhagen and V. Braun, Superconformal field theories for compact manifolds with spin(7) holonomy, JHEP 12 (2001) 013 [hep-th/0111048] [INSPIRE].

[26] B. de Wit, I. Herger and H. Samtleben, Gauged locally supersymmetric $D=3$ nonlinear o-models, Nucl. Phys. B 671 (2003) 175 [hep-th/0307006] [INSPIRE].

[27] B. de Wit, H. Nicolai and H. Samtleben, Gauged supergravities in three-dimensions: a panoramic overview, hep-th/0403014 [INSPIRE].

[28] D. Martelli and J. Sparks, G structures, fluxes and calibrations in M-theory, Phys. Rev. D 68 (2003) 085014 [hep-th/0306225] [INSPIRE]. 
[29] D. Tsimpis, $M$-theory on eight-manifolds revisited: $N=1$ supersymmetry and generalized spin(7) structures, JHEP 04 (2006) 027 [hep-th/0511047] [INSPIRE].

[30] G. Gibbons, D.N. Page and C. Pope, Einstein Metrics on $S^{3} R^{3}$ and $R^{4}$ Bundles, Commun. Math. Phys. 127 (1990) 529 [INSPIRE].

[31] T.W. Grimm and J. Louis, The effective action of type IIA Calabi-Yau orientifolds, Nucl. Phys. B 718 (2005) 153 [hep-th/0412277] [INSPIRE].

[32] O. Hohm and J. Louis, Spontaneous $N=2 \rightarrow N=1$ supergravity breaking in three-dimensions, Class. Quant. Grav. 21 (2004) 4607 [hep-th/0403128] [INSPIRE].

[33] D. Forcella and A. Zaffaroni, $N=1$ Chern-Simons theories, orientifolds and Spin(7) cones, JHEP 05 (2010) 045 [arXiv:0911.2595] [INSPIRE].

[34] M. Haack and J. Louis, Duality in heterotic vacua with four supercharges, Nucl. Phys. B 575 (2000) 107 [hep-th/9912181] [INSPIRE].

[35] M. Haack and J. Louis, M theory compactified on Calabi-Yau fourfolds with background flux, Phys. Lett. B 507 (2001) 296 [hep-th/0103068] [INSPIRE].

[36] T.W. Grimm and H. Hayashi, F-theory fluxes, chirality and Chern-Simons theories, JHEP 03 (2012) 027 [arXiv:1111.1232] [inSPIRE].

[37] M. Cvetič, T.W. Grimm and D. Klevers, Anomaly cancellation and Abelian gauge symmetries In F-theory, JHEP 02 (2013) 101 [arXiv:1210.6034] [INSPIRE].

[38] M. Cvetič, A. Grassi, D. Klevers and H. Piragua, Chiral Four-Dimensional F-theory Compactifications With SU(5) and Multiple U(1)-Factors, arXiv:1306.3987 [INSPIRE].

[39] T.W. Grimm and R. Savelli, Gravitational Instantons and Fluxes from M/F-theory on Calabi-Yau fourfolds, Phys. Rev. D 85 (2012) 026003 [arXiv:1109.3191] [InSPIRE].

[40] S. Weinberg, Photons and Gravitons in s Matrix Theory: Derivation of Charge Conservation and Equality of Gravitational and Inertial Mass, Phys. Rev. 135 (1964) B1049.

[41] M.T. Grisaru and H. Pendleton, Soft Spin 3/2 Fermions Require Gravity and Supersymmetry, Phys. Lett. B 67 (1977) 323 [INSPIRE].

[42] F. Bonetti and T.W. Grimm, Six-dimensional $(1,0)$ effective action of F-theory via M-theory on Calabi-Yau threefolds, JHEP 05 (2012) 019 [arXiv:1112.1082] [INSPIRE].

[43] A. Sen, Orientifold limit of F-theory vacua, Phys. Rev. D 55 (1997) 7345 [hep-th/9702165] [INSPIRE].

[44] M. Graña and H. Triendl, Generalized $N=1$ and $N=2$ structures in $M$-theory and type-II orientifolds, JHEP 03 (2013) 145 [arXiv:1211.3867] [INSPIRE].

[45] I. Antoniadis, E. Dudas and A. Sagnotti, Brane supersymmetry breaking, Phys. Lett. B 464 (1999) 38 [hep-th/9908023] [INSPIRE].

[46] O. Hohm, Massive Kaluza-Klein theories and their spontaneously broken symmetries, hep-th/0612235 [INSPIRE].

[47] F. Bonetti, T.W. Grimm and S. Hohenegger, A Kaluza-Klein inspired action for chiral p-forms and their anomalies, Phys. Lett. B 720 (2013) 424 [arXiv:1206.1600] [INSPIRE].

[48] M. Esole, J. Fullwood and S.-T. Yau, $D_{5}$ elliptic fibrations: non-Kodaira fibers and new orientifold limits of F-theory, arXiv:1110.6177 [INSPIRE]. 